LPT-Orsay 05/31

DESY 05-097

ULB-TH/05-16

FTUAM 05/9

IFT-UAM/CSIC-05-29

\title{
Adiabatic compression and indirect detection of supersymmetric dark matter
}

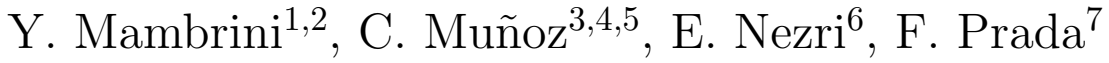 \\ ${ }^{1}$ Laboratoire de Physique Théorique, Université Paris-Sud, F-91405 Orsay, France \\ 2 Deutsches Elektronen-Synchrotron DESY, Notkestrasse 85, 22607 Hamburg, Germany \\ ${ }^{3}$ Departamento de Física Teórica C-XI, Universidad Autónoma de Madrid, \\ Cantoblanco, 28049 Madrid, Spain \\ ${ }^{4}$ Instituto de Física Teórica C-XVI, Universidad Autónoma de Madrid, \\ Cantoblanco, 28049 Madrid, Spain \\ ${ }^{5}$ Department of Physics, Korea Advanced Institute of Science and Technology, \\ Daejeon 305-701, Korea \\ ${ }^{6}$ Service de Physique Théorique, CP225, Université Libre de Bruxelles, \\ 1050 Bruxelles, Belgium \\ ${ }^{7}$ Instituto de Astrofísica de Andalucía (CSIC), E-18008 Granada, Spain
}

\begin{abstract}
Recent developments in the modelling of the dark matter distribution in our Galaxy point out the necessity to consider some physical processes to satisfy observational data. In particular, models with adiabatic compression, which include the effect of the baryonic gas in the halo, increase significantly the dark matter density in the central region of the Milky Way. On the other hand, the non-universality in scalar and gaugino sectors of supergravity models can also increase significantly the neutralino annihilation cross section. We show that the combination of both effects gives rise to a gamma-ray flux arising from the galactic center largely reachable by future experiments like GLAST. We also analyse in this framework the EGRET excess data above $1 \mathrm{GeV}$, as well as the recent data from CANGAROO and HESS. The analysis has been carried out imposing the most recent experimental constraints, such as the lower bound on the Higgs mass, the $b \rightarrow s \gamma$ branching ratio, and the muon $g-2$. In addition, the recently improved upper bound on $B\left(B_{s} \rightarrow \mu^{+} \mu^{-}\right)$has also been taken into account. The astrophysical (WMAP) bounds on the dark matter density have also been imposed on the theoretical computation of the relic neutralino density through thermal production.
\end{abstract}




\section{Contents}

1 Introduction $\quad 3$

2 Astrophysical inputs and adiabatic compression 4

2.1 Gamma-ray flux . . . . . . . . . . . . . . . 5

2.2 Halo models . . . . . . . . . . . . . . . . . . . . . . . . 6

3 Neutralino dark matter $\quad 9$

3.1 Supergravity models . . . . . . . . . . . . . . . . . . . . 9 9

3.2 Experimental and astrophysical constraints . . . . . . . . . . . . 11

4 Comparison with the experiments $\quad 15$

4.1 EGRET . . . . . . . . . . . . . . . . . . . 15

4.2 GLAST . . . . . . . . . . . . . . . . . 18

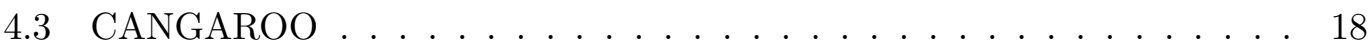

4.4 Combining EGRET and CANGAROO . . . . . . . . . . . 22

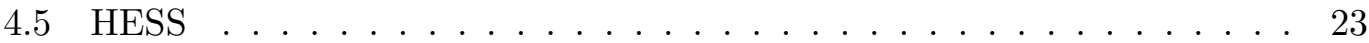

5 Conclusions $\quad 27$ 


\section{Introduction}

It is now well established that luminous matter makes up only a small fraction of the mass observed in Universe. A weakly interacting massive particle (WIMP) is one of the leading candidates for the "dark" component of the Universe. Indeed, WIMPs may be present in the right amount to explain the matter density observed in the analysis of galactic rotation curves [1], cluster of galaxies, and large scale flows [2], implying $0.1 \lesssim \Omega_{D M} h^{2} \lesssim 0.3$. Actually, the recent data obtained by the WMAP satellite [3] confirm that dark matter is present, and they lead to the value $0.094 \lesssim \Omega_{D M} h^{2} \lesssim 0.129$.

One of the most promising methods for the indirect detection of WIMPs consists of detecting the gamma rays produced by their annihilations in the galactic halo [4][30]. The amount of gamma-ray fluxes observed will depend of course on the nature of WIMPs through their annihilation cross sections, but also on their density. This is the reason why the inner center of our galaxy $(\sim 100 \mathrm{pc}$ from the center), where the dark matter density is large, is the main theater of dark matter searches through gamma-ray signatures. Atmospheric Cherenkov telescopes or space-based detectors are used for this search. For example, one of the space-based experiments, EGRET, detected a signal [31] that apparently is difficult to explain with the usual gamma-ray background. Also, the atmospheric telescope CANGAROO-II claims a significant detection of gamma rays from the galactic center region [32]. In addition, the atmospheric telescope HESS has also observed gamma-rays [33] in Sagittarius A* direction. Projected experiments might clarify the situation. This is the case of the space telescope GLAST [34], which is scheduled for launch in 2006, and will be able to provide a larger sensitivity.

Concerning the nature of WIMPs, the best motivated candidate is the lightest neutralino, a particle predicted by the supersymmetric (SUSY) extension of the standard model [35]. In particular, in most of the parameter space of the minimal supersymmetric standard model (MSSM) the lightest supersymmetric particle (LSP) is the lightest neutralino. Thus it is absolutely stable and therefore a candidate for dark matter. In addition, the neutralino is an electrically neutral particle, and this is welcome since otherwise it would bind to nuclei and would be excluded as a candidate for dark matter from unsuccessful searches for exotic heavy isotopes.

Recently, SUSY dark matter candidates have been studied in the context of realistic halo models including baryonic matter [36]. Indeed, since the total mass of the inner galaxy is dominated by baryons, the dark matter distribution is likely to have been influenced by the baryonic potential. In particular, its density is increased, and as a consequence typical halo profiles such as Navarro, Frenk and White (NFW) [37] and Moore et al. [38] have a more singular behaviour near the galactic center. The conclusion of the work in Ref. [36] is that the gamma-ray flux produced by the annihilation of neutralinos in the galactic center is increased significantly, and is within the sensitivity of projected experiments, when density profiles with baryonic compression are taken into account.

Nevertheless, this result has been obtained assuming particular values for the neutralino mass and annihilation cross sections $\sigma_{i}$, and therefore a more detailed analysis computing explicitly $\sigma_{i}$ in the framework of SUSY must be carried out. It has also 
been discussed recently that another way of increasing the gamma-ray flux produced by the annihilation of neutralinos is to consider the possibility of non-universality in the scalar and gaugino sectors of the MSSM [20, 21, 26, 28]. In particular, in the context of supergravity (SUGRA) the flux can be increased significantly. This is so when departures from the minimal supergravity (mSUGRA) scenario, where the soft terms of the MSSM are assumed to be universal at the unification scale and radiative electroweak symmetry breaking is imposed, are taken into account. Using non-universal soft scalar and gaugino masses the annihilation cross section of neutralinos can be increased, producing a larger gamma-ray flux [26, 28].

The aim of this work is to discuss in detail both effects, halo models with baryonic compression and non-universal soft terms in SUGRA, on the gamma-ray flux. We will see that a significant enhancement is obtained, and regions of the SUGRA parameter space are compatible with the sensitivity of present and projected experiments.

Let us remark that in addition to the astrophysical bounds on the dark matter density discussed above, the most recent experimental constraints are also considered in the analysis. In particular, we implement the lower bounds on the masses of SUSY particles and Higgs boson, as well as the experimental bounds on the branching ratio of the $b \rightarrow s \gamma$ process and on $a_{\mu}^{\mathrm{SUSY}}$, for which the more stringent constraint from $e^{+} e^{-}$ disfavors important regions of the SUGRA parameter space (see e.g. Ref. $[23,26]$ ). In addition, we have also taken into account the last data concerning the $B_{s} \rightarrow \mu^{+} \mu^{-}$ branching ratio. It is now known that the upper bound on this process constrains strongly the parameter space of non-universal SUGRA models, and has important implications for direct searches of dark matter $[39,40]$. Thus an analysis in the case of indirect detection is necessary and has been carried out here.

The paper is organized as follows. In Section 2 we will discuss in general the gammaray detection from dark matter annihilation, paying special attention to its halo model dependence. In particular, the modifications produced in the different profiles due to the effects of baryonic compression will be studied. The particle physics dependence will be reviewed in Section 3 for the case of neutralinos in SUGRA. We will study the general case when scalar and gaugino non-universalities are present. In addition, the most recent experimental and astrophysical constraints will be taken into account in the discussion. In Section 4 both effects, halo models with baryonic compression and non-universal soft terms, will be considered for the study of the gamma-ray flux. The theoretical predictions will be compared with the sensitivity of present and projected experiments, such as EGRET, CANGAROO, HESS, and GLAST. Finally, the conclusions are left for Section 5.

\section{Astrophysical inputs and adiabatic compres- sion}

As discussed in the Introduction, we are interested in the annihilation of dark matter particles in the galactic center [5]. There are two possible types of gamma rays that can be produced by the annihilation. First, gamma-ray lines from processes $\chi \chi \rightarrow \gamma \gamma$ $[10]$ and $\chi \chi \rightarrow \gamma Z$ [11]. This signal would be very clear since the photons are basically 
mono-energetic. Unfortunately, the neutralino does not couple directly to the photon, the Feynman diagrams are loop suppressed, and therefore the flux would be small [12]. For more recent analyses of this possibility see Refs. [9, 17, 26].

On the other hand, continuum gamma rays produced by the decay of neutral pions generated in the cascading of annihilation products will give rise to larger fluxes [13]. We will concentrate on this possibility in the following.

Let us then discuss the theoretical formulas for the computation of the continuum gamma rays, and in particular the astrophysical inputs one has to use. As we will see below, the gamma-ray flux can be significantly enhanced for specific dark matter density profiles.

\subsection{Gamma-ray flux}

For the continuum of gamma rays, the observed differential flux at the Earth coming from a direction forming an angle $\psi$ with respect to the galactic center is

$$
\Phi_{\gamma}\left(E_{\gamma}, \psi\right)=\sum_{i} \frac{d N_{\gamma}^{i}}{d E_{\gamma}}\left\langle\sigma_{i} v\right\rangle \frac{1}{8 \pi m_{\chi}^{2}} \int_{\text {line of sight }} \rho^{2} d l,
$$

where the discrete sum is over all dark matter annihilation channels, $d N_{\gamma}^{i} / d E_{\gamma}$ is the differential gamma-ray yield, $\left\langle\sigma_{i} v\right\rangle$ is the annihilation cross section averaged over its velocity distribution, $m_{\chi}$ is the mass of the dark matter particle, and $\rho$ is the dark matter density. Assuming a spherical halo, one has $\rho=\rho(r)$ with the galactocentric distance $r^{2}=l^{2}+R_{0}^{2}-2 l R_{0} \cos \psi$, where $R_{0}$ is the solar distance to the galactic center $(\simeq 8 \mathrm{kpc})$. It is customary to separate in the above equation the particle physics part from the halo model dependence introducing the (dimensionless) quantity

$$
J(\psi)=\frac{1}{8.5 \mathrm{kpc}}\left(\frac{1}{0.3 \mathrm{GeV} / \mathrm{cm}^{3}}\right)^{2} \int_{\text {line of sight }} \rho^{2}(r(l, \psi)) d l .
$$

Thus one can write

$$
\begin{aligned}
\Phi_{\gamma}\left(E_{\gamma}, \psi\right) & =0.94 \times 10^{-13} \mathrm{~cm}^{-2} \mathrm{~s}^{-1} \mathrm{GeV}^{-1} \mathrm{sr}^{-1} \\
& \times \sum_{i} \frac{d N_{\gamma}^{i}}{d E_{\gamma}}\left(\frac{\left\langle\sigma_{i} v\right\rangle}{10^{-29} \mathrm{~cm}^{3} \mathrm{~s}^{-1}}\right)\left(\frac{100 \mathrm{GeV}}{m_{\chi}}\right)^{2} J(\psi) .
\end{aligned}
$$

Actually, when comparing to experimental data one must consider the integral of $J(\psi)$ over the spherical region of solid angle $\Delta \Omega$ given by the angular acceptance of the detector which is pointing towards the galactic center, i.e. the quantity $\bar{J}(\Delta \Omega)$ with

$$
\bar{J}(\Delta \Omega) \equiv \frac{1}{\Delta \Omega} \int_{\Delta \Omega} J(\psi) d \Omega
$$

must be used in the above equation. For example, for EGRET $\Delta \Omega$ is about $10^{-3} \mathrm{sr}$ whereas for GLAST, CANGAROO, and HESS it is $10^{-5} \mathrm{sr}$. 
The gamma-ray flux can now be expressed as

$$
\begin{aligned}
\Phi_{\gamma}\left(E_{t h r}\right) & =0.94 \times 10^{-13} \mathrm{~cm}^{-2} \mathrm{~s}^{-1} \\
& \times \sum_{i} \int_{E_{t h r}}^{m_{\chi}} d E_{\gamma} \frac{d N_{\gamma}^{i}}{d E_{\gamma}}\left(\frac{\left\langle\sigma_{i} v\right\rangle}{10^{-29} \mathrm{~cm}^{3} \mathrm{~s}^{-1}}\right)\left(\frac{100 \mathrm{GeV}}{m_{\chi}}\right)^{2} \bar{J}(\Delta \Omega) \Delta \Omega,
\end{aligned}
$$

where $E_{t h r}$ is the lower threshold energy of the detector, Concerning the upper limit of the integral, note that neutralinos move at galactic velocity and therefore their annihilation occurs at rest.

\subsection{Halo models}

A crucial ingredient for the calculation of $\bar{J}$ in (2.4), and therefore for the calculation of the flux of gamma rays, is the dark matter density profile of our galaxy. The different profiles that have been proposed in the literature can be parameterised as [41]

$$
\rho(r)=\frac{\rho_{0}\left[1+\left(R_{0} / a\right)^{\alpha}\right]^{(\beta-\gamma) / \alpha}}{\left(r / R_{0}\right)^{\gamma}\left[1+(r / a)^{\alpha}\right]^{(\beta-\gamma) / \alpha}},
$$

where $\rho_{0}$ is the local (solar neighborhood) halo density and $a$ is a characteristic length. Although we will use $\rho_{0}=0.3 \mathrm{GeV} / \mathrm{cm}^{3}$ throughout the paper, since this is just a scaling factor in the analysis, modifications to its value can be straightforwardly taken into account in the results. Highly cusped profiles are deduced from $N$-body simulations ${ }^{1}$. In particular, NFW [37] obtained a profile with a behaviour $\rho(r) \propto r^{-1}$ at small distances. A more singular behaviour, $\rho(r) \propto r^{-1.5}$, was obtained by Moore et al. [38]. However, these predictions are valid only for halos without baryons. One can improve simulations in a more realistic way by taking into account the effect of the normal gas (baryons). This loses its energy through radiative processes falling to the central region of forming galaxy. As a consequence of this redistribution of mass, the resulting gravitational potential is deeper, and the dark matter must move closer to the center increasing its density.

This increase in the dark matter density is often treated using adiabatic invariants. The present form of the adiabatic compression model was numerically and analytically studied by Blumental et al. [43]. This model assumes spherical symmetry, circular orbit for the particles, and conservation of the angular momentum $M(r) r=$ const., where $M(r)$ is the total mass enclosed within radius $r$. The mass distributions in the initial and final configurations are therefore related by $M_{i}\left(r_{i}\right) r_{i}=\left[M_{b}\left(r_{f}\right)+M_{D M}\left(r_{f}\right)\right] r_{f}$, where $M_{i}(r), M_{b}(r)$ and $M_{D M}(r)$ are the mass profile of the galactic halo before the cooling of the baryons (obtained through $N$-body simulations), the baryonic composition of the Milky Way observed now, and the to be determined dark matter component of the halo today, respectively. This approximation was tested in numerical simulations $[44,45]$. Nevertheless, a more precise approximation can be obtained including the possibility of elongated orbits [36]. In this case, the mass inside the orbit, $M(r)$, is smaller than the real mass, the one the particle 'feels' during its revolution around

\footnotetext{
${ }^{1}$ For analytical derivations see e.g. the recent work [42], and references therein.
} 
the galactic center. As a consequence, the modified compression model is based on the conservation of the product $M(\bar{r}) r$, where $\bar{r}$ is the averaged radius of the orbit. The time average radii is given by $\bar{x} \sim 1.72 x^{0.82} /(1+5 x)^{0.085}$, with $x \equiv r / r_{s}$, and $r_{s}$ the characteristic radius of the assumed approximation.

The models and constraints that we used in this work for the Milky Way can be found in Table I of Ref. [36]. We have fitted the resulting data of that work with the power-law parameterisation of eq. (2.6). The results are shown in Fig. 1, and summarized in Table 1. There we label the resulting NFW and Moore et al. profiles with adiabatic compression by $\mathrm{NFW}_{\mathrm{c}}$ and $\mathrm{Moore}_{\mathrm{c}}$, respectively. As one can see, at small $r$ the dark matter density profile following the adiabatic cooling of the baryonic fraction is a steep power law $\rho \propto r^{-\gamma_{c}}$ with $\gamma_{c} \approx 1.45(1.65)$ for a $\mathrm{NFW}_{\mathrm{c}}\left(\right.$ Moore $_{\mathrm{c}}$ ) compressed model $^{2}$. We observe for example that the effect of the adiabatic compression on a NFW profile is basically to transform it into a Moore et al. one (see Table 1).

Let us remark that NFW and Moore et al. profiles can be considered as a lower and upper limit, respectively. For example, the one recently proposed by Diemand, Moore and Stadel in Ref. [46], where $\rho(r) \propto r^{-1.16}$, is between both 'standard' profiles. It will be interesting for the analysis in Sect. 4 to use an average value of $\bar{J}$ for compressed scenarios. In particular, we will consider a value $\bar{J}^{\prime}=5 \times \bar{J}_{\mathrm{NFW}_{\mathrm{c}}}$, and we will refer to this scenario as $\mathrm{NFW}_{\mathrm{c}}^{\prime}$ for simplicity. It is worth noticing in this sense than some mechanisms can be advocated to reduce the effect of the compression like angular momentum transfer to dark matter or formation of the central black hole by spiraling and merging of two black holes. The study of such complex mechanisms is far beyond the scope of this work.

Of course, these results have important implications for the computation of the gamma-ray fluxes from the galatic center. In particular, in Table 1 we see that for $\Delta \Omega=$ $10^{-3}$ sr one has $\bar{J}_{\mathrm{NFW}_{\mathrm{c}}} / \bar{J}_{\mathrm{NFW}} \simeq 145$ and $\bar{J}_{\text {Moore }_{\mathrm{c}}} / \bar{J}_{\text {Moore et al. }} \simeq 77$. Thus the effect of the adiabatic compression is very strong, increasing the gamma-ray fluxes about two orders of magnitude. Similarly, for $\Delta \Omega=10^{-5}$ sr one obtains $\bar{J}_{\mathrm{NFW}_{\mathrm{c}}} / \bar{J}_{\mathrm{NFW}} \simeq 953$ and $\bar{J}_{\text {Moorec }} / \bar{J}_{\text {Moore et al. }} \simeq 95$.

Let us finally remark that the $\bar{J}$ calculation has been regulated by assuming a constant density for $r<10^{-5} \mathrm{kpc}$. This procedure has consequences essentially for divergent $\bar{J}$ when $\gamma \geq 1.5$. To check our results we have also calculated $\bar{J}$ in the following way. We used a cut off in density, $\rho\left(r<R_{s}\right)=0, R_{s}$ being the Schwarchild radius of a $3 \times 10^{6} M_{\odot}$ black hole. In addition we also considered dark matter annihilation and estimate its effect by requiring an upper value for dark matter density, $\rho\left(r<r_{\max }\right)=\rho\left(r_{\max }\right)=m_{\chi} /\left(\langle\sigma v\rangle \cdot t_{B H}\right)\left(t_{B H} \sim 10^{10} \mathrm{yr}\right.$, being the age of the black hole). We also applied the same procedure with a more realistic cut-off value $\rho\left(r<10^{-6} \mathrm{pc}\right)=0$ suggested by dark matter particle scatterings on stars [47]. This kind of effects can be significant only for the Moore $_{c}$ halo model we consider in this paper and increase our $\bar{J}$ value by less than an order of magnitude for all the $\langle\sigma v\rangle$ values corresponding to our wide SUSY parameter range. In this sense our analysis is conservative. More refine treatments of dark matter annihilation effects in the innermost region of the galaxy can be found in $[48,49]$.

\footnotetext{
${ }^{2}$ It is worth noticing that we obtain the same order of magnitude as the authors of Ref. [48].
} 

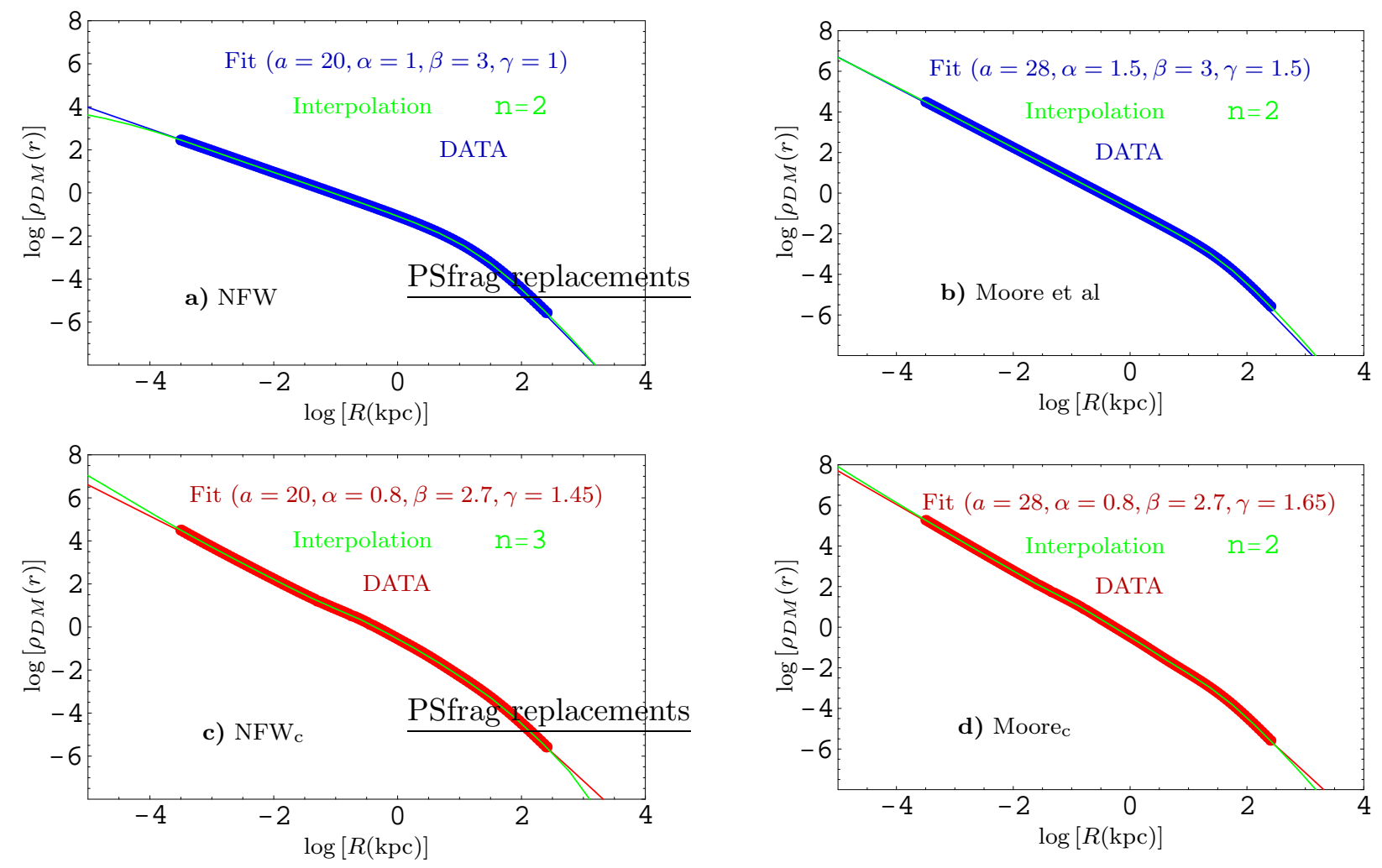

Figure 1: Fit, interpolation and data for: a) NFW, b) Moore et al., c) NFW compressed, and d) Moore et al. compressed .

\begin{tabular}{|c|cccccc|}
\hline & $\mathrm{a}(\mathrm{kpc})$ & $\alpha$ & $\beta$ & $\gamma$ & $\bar{J}\left(10^{-3} \mathrm{sr}\right)$ & $\bar{J}\left(10^{-5} \mathrm{sr}\right)$ \\
\hline $\mathrm{NFW}$ & 20 & 1 & 3 & 1 & $1.214 \times 10^{3}$ & $1.264 \times 10^{4}$ \\
NFW $_{\mathrm{c}}$ & 20 & 0.8 & 2.7 & 1.45 & $1.755 \times 10^{5}$ & $1.205 \times 10^{7}$ \\
Moore et al. & 28 & 1.5 & 3 & 1.5 & $1.603 \times 10^{5}$ & $5.531 \times 10^{6}$ \\
Moore $_{\mathrm{c}}$ & 28 & 0.8 & 2.7 & 1.65 & $1.242 \times 10^{7}$ & $5.262 \times 10^{8}$ \\
\hline
\end{tabular}

Table 1: NFW and Moore et al. density profiles without and with adiabatic compression $\left(\mathrm{NFW}_{\mathrm{c}}\right.$ and Moore $_{\mathrm{c}}$ respectively) with the corresponding parameters, and values of $\bar{J}(\Delta \Omega)$. 

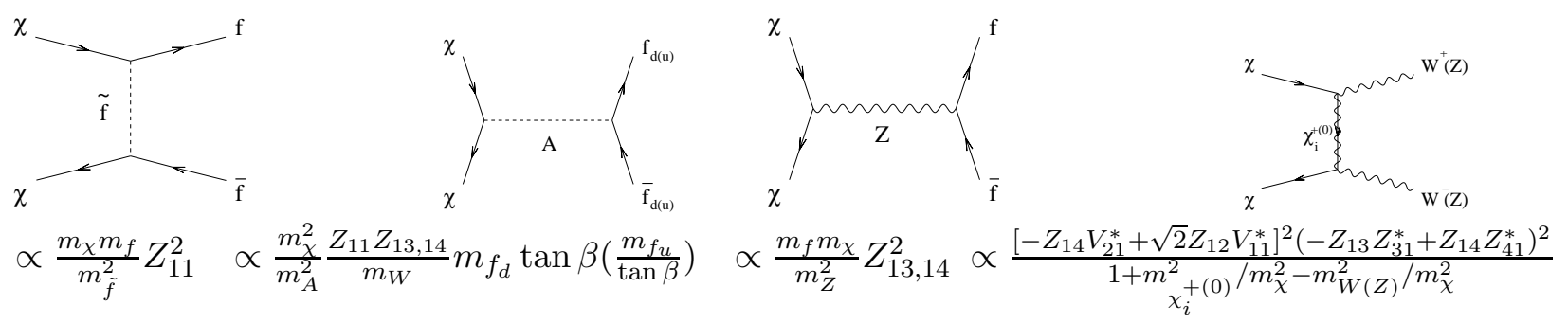

Figure 2: Dominant neutralino annihilation diagrams. Relevant parts of the amplitudes are shown explicitly Terms between parenthesis correspond to $f_{u}$ and $Z$ final states in second and fourth diagrams. $\mathrm{V}$ and $\mathrm{Z}$ are chargino and neutralino mixing matrices.

\section{Neutralino dark matter}

For the computation of the gamma-ray flux discussed in the previous section, in addition to the halo profile it is also crucial the value of the annihilation cross section $\sigma_{i}$. Of course, for determining this value the theoretical framework must be established.

As mentioned in the Introduction, we will work in the context of the MSSM. Let us recall that in this framework there are four neutralinos, $\tilde{\chi}_{i}^{0}(i=1,2,3,4)$, since they are the physical superpositions of the fermionic partners of the neutral electroweak gauge bosons, called bino $\left(\tilde{B}^{0}\right)$ and wino $\left(\tilde{W}_{3}^{0}\right)$, and of the fermionic partners of the neutral

Higgs bosons, called Higgsinos $\left(\tilde{H}_{u}^{0}, \tilde{H}_{d}^{0}\right)$. Thus one can express the lightest neutralino as

$$
\tilde{\chi}_{1}^{0}=Z_{11} \tilde{B}^{0}+Z_{12} \tilde{W}_{3}^{0}+Z_{13} \tilde{H}_{d}^{0}+Z_{14} \tilde{H}_{u}^{0} .
$$

It is commonly defined that $\tilde{\chi}_{1}^{0}$ is mostly gaugino-like if $P \equiv\left|Z_{11}\right|^{2}+\left|Z_{12}\right|^{2}>0.9$, Higgsino-like if $P<0.1$, and mixed otherwise.

In figure 2 we show the relevant Feynman diagrams contributing to neutralino annihilation. As was remarked e.g. in Ref. [26, 50, 51, 21], the annihilation cross section can be significantly enhanced depending on the SUSY model under consideration. We will concentrate here on the SUGRA scenario, where the soft terms are determined at the unification scale, $M_{G U T} \approx 2 \times 10^{16} \mathrm{GeV}$, after SUSY breaking, and radiative electroweak symmetry breaking is imposed.

\subsection{Supergravity models}

Let us discuss first the mSUGRA scenario, where the soft terms of the MSSM are assumed to be universal at $M_{G U T}$. Recall that in mSUGRA one has only four free parameters: the soft scalar mass $m$, the soft gaugino mass $M$, the soft trilinear coupling $A$, and the ratio of the Higgs vacuum expectation values, $\tan \beta=\left\langle H_{u}^{0}\right\rangle /\left\langle H_{d}^{0}\right\rangle$. In addition, the sign of the Higgsino mass parameter, $\mu$, remains also undetermined by the minimization of the Higgs potential.

Since in this scenario the lightest neutralino $\tilde{\chi}_{1}^{0}$ is mainly bino, only $Z_{11}$ is large and then the contribution of diagrams in Fig. 2 will be generically small, the first being 
suppressed by $\tilde{f}$ mass. As a consequence, the predicted annihilation cross section $\sigma_{i}$ is small, and therefore the flux is generically below the present accessible experimental regions (unless $\tan \beta \gtrsim 50$ combined with a Moore et al. profile are considered)

However, as discussed in detail in Ref. [26] in the context of indirect detection, $\sigma_{i}$ can be increased in different ways when the structure of mSUGRA for the soft terms is abandoned. In particular, it is possible to enhance the annihilation channels involving exchange of the CP-odd Higgs, $A$, by reducing the Higgs mass. In addition, it is also possible to increase the Higgsino components of the lightest neutralino $Z_{13,14}$. Thus annihilation channels through Higgs exchange become more important than in mSUGRA. This is also the case for $Z^{-}, \chi_{1}^{ \pm}$, and $\tilde{\chi}_{1}^{0}$-exchange channels. As a consequence, the gamma-ray flux will be increased.

In particular, the most important effects are produced by the non-universality of Higgs and gaugino masses. These can be parameterised, at $M_{G U T}$, as follows

$$
m_{H_{d}}^{2}=m^{2}\left(1+\delta_{1}\right), \quad m_{H_{u}}^{2}=m^{2}\left(1+\delta_{2}\right),
$$

and

$$
M_{1}=M, \quad M_{2}=M\left(1+\delta_{2}^{\prime}\right), \quad M_{3}=M\left(1+\delta_{3}^{\prime}\right),
$$

We will concentrate in our analysis on the following representative cases:

$$
\begin{aligned}
& \text { a) } \delta_{1}=0 \quad, \delta_{2}=0 \quad, \delta_{2,3}^{\prime}=0 \text {, } \\
& \text { b) } \delta_{1}=0 \quad, \delta_{2}=1 \quad, \delta_{2,3}^{\prime}=0 \text {, } \\
& \text { c) } \delta_{1}=-1, \delta_{2}=0 \quad, \delta_{2,3}^{\prime}=0 \text {, } \\
& \text { d) } \delta_{1}=-1, \delta_{2}=1 \quad, \delta_{2,3}^{\prime}=0 \text {, } \\
& \text { e) } \delta_{1,2}=0 \quad, \delta_{2}^{\prime}=0 \quad, \delta_{3}^{\prime}=-0.5 \text {, } \\
& \text { f) } \delta_{1,2}=0 \quad, \delta_{2}^{\prime}=-0.5 \quad, \delta_{3}^{\prime}=0 \text {. }
\end{aligned}
$$

Case a) corresponds to mSUGRA with universal soft terms, cases b), c) and d) correspond to non-universal Higgs masses, and finally cases e) and f) to non-universal gaugino masses. The cases $b$ ), c), d), and e) were discussed in Ref. [26], and are known to produce gamma-ray fluxes larger than in mSUGRA, whereas case $f$ ) will be of interest when discussing heavy WIMP signals predictions in the perspective of atmospheric Cherenkov telescopes like e.g. CANGAROO.

We will discuss all these cases taking into account in the computation of the gammaray flux the effect of the adiabatic compression. Except for the calculation of the $\bar{J}$ factor, for the evaluation of the fluxes we used the last DarkSusy released version [52]. To solve the renormalization group equations for the soft SUSY-breaking terms between $M_{G U T}$ and the electroweak scale, we used the Fortran package SUSPECT [53].

Depending on the non-universal case, the main parameters that enter in the flux computation, namely the neutralino mass $m_{\chi}$ and the annihilation rate $N_{\gamma}\langle\sigma v\rangle \equiv$ $\sum_{i} \int_{E_{t h r}}^{m_{\chi}} d E_{\gamma} \frac{d N_{\gamma}^{i}}{d E_{\gamma}}\left\langle\sigma_{i} v\right\rangle$ can vary significantly within the parameter space. Running a scan on the gaugino mass parameter $M$ between 0 and $2 \mathrm{TeV}$, we expect a neutralino mass lying between $200 \mathrm{GeV}$ and $1 \mathrm{TeV}$, the lower bound being mainly restricted by accelerator constraints on the chargino or lighter higgs mass. We can however reach 
3 to $4 \mathrm{TeV}$ neutralinos respecting all the experimental and cosmological constraints if we extend the range of the scanning on $M$ to $10 \mathrm{TeV}$. On the other hand, the WMAP relic density constraint restricts the annihilation cross section $N_{\gamma}\langle\sigma v\rangle$ to lie from $\sim$ $1 \times 10^{-28} \mathrm{~cm}^{3} \mathrm{~s}^{-1}$ (when the coannihilation process with the lighter stau dominates, cases $a$ ) or $c$ ) with low $\tan \beta$ ) to $\sim 2 \times 10^{-26} \mathrm{~cm}^{3} \mathrm{~s}^{-1}$ (when the $A$-pole region dominates, case $c$ ), d) and $e$ ) with $\tan \beta=35)^{3}$.

Before carrying out the analysis in detail, it is worth remarking that in these studies it is crucial to reproduce the correct phenomenology. Thus, in the next section, the most recent experimental and astrophysical constraints which can affect the computation will be discussed.

\subsection{Experimental and astrophysical constraints}

We have taken into account in the computation the most recent experimental and astrophysical bounds. These may produce important constraints on the parameter space of SUGRA models, restricting therefore the regions where the gamma-ray flux has to be analyzed.

In particular, concerning the astrophysical bounds, the effect of $0.1 \lesssim \Omega_{D M} h^{2} \lesssim 0.3$, on the relic neutralino density computation has been considered. Due to its relevance, the WMAP narrow range $0.094 \lesssim \Omega_{D M} h^{2} \lesssim 0.129$ has also been analyzed in detail. Actually, we have also considered the possibility that not all the dark matter is made of neutralinos, allowing $0.03<\Omega_{\tilde{\chi}_{1}^{0}} h^{2}<0.094$. In this case we have to rescale the density of neutralinos in the galaxy $\rho(r)$ in Eq. (2.6) by a factor $\Omega_{\tilde{\chi}_{1}^{0}} h^{2} / 0.094$.

We illustrate this effect in Fig. 3 for the case of mSUGRA, i.e. case $a$ ) of (3.10). There, the cosmologically allowed regions are shown in the parameter space $(m, M)$ for $\tan \beta=50, A=0$ and $\mu>0$. As discussed above, in most of the parameter space the lightest neutralino is mainly bino, and as a consequence the annihilation cross section is small producing a too large relic abundance. Nevertheless, let us recall that there are basically four corridors where the above bounds can be satisfied. There is the narrow coannihilation branch of the parameter space, i.e. the region where the stau is the next to the LSP producing efficient coannihilations (for ${ }^{4} 500 \lesssim m_{\tilde{\chi}_{1}^{0}} \lesssim 800 \mathrm{GeV}$ ). This reduces considerably the relic abundance and place it inside the bounds. Point A in the figure corresponds to this situation. Nevertheless, the neutralino annihilation in the galactic center is dominated by the $A$-Higgs exchange, but being far from the $A$-pole region $\left(2 m_{\tilde{\chi}_{1}^{0}} \sim m_{A}\right)$ the gamma-ray flux is not very large compared with the ones produced in the other regions to be discussed below (See Fig. 6a below, where this narrow corridor can be clearly distinguished).

Now, for a given value of $m$, and from large to small $M, m_{A}$ decreases. When $m_{A}$ becomes close to $2 m_{\tilde{\chi}_{1}^{0}}$ the annihilation cross section increases and therefore the relic density decreases entering in an allowed corridor (see e.g. point B) with $0.094<\Omega_{\tilde{\chi}_{1}^{0}} h^{2}<$

\footnotetext{
${ }^{3}$ In some fine tuned regions of parameter space with especially large values of $m$ (Focus Point region) it is possible to reach $\langle\sigma v\rangle \sim 10^{-25} \mathrm{~cm}^{3} \mathrm{~s}^{-1}$ in the cases b), d) and $e$ )

${ }^{4}$ Recall that we can deduce these values in the plot from the value of $M$, since in $\mathrm{mSUGRA} m_{\tilde{\chi}_{1}^{0}} \simeq M_{1} \simeq$ $0.4 M$
} 


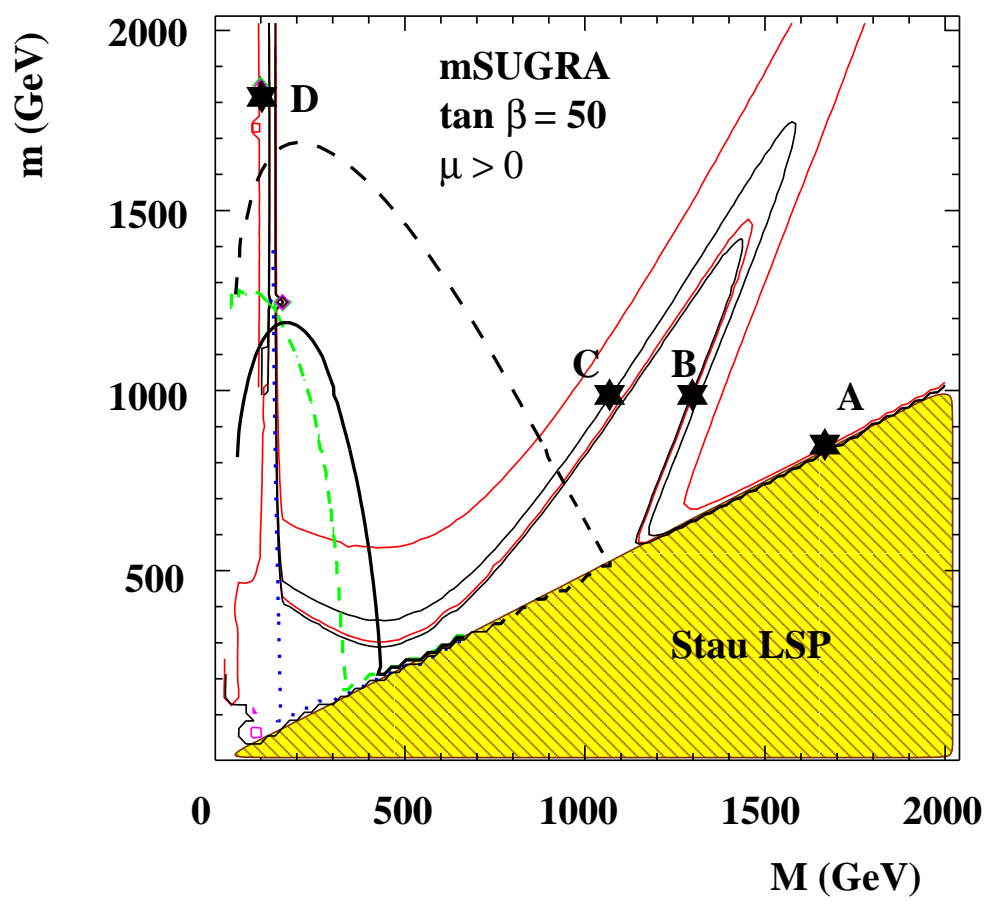

Figure 3: Experimental and astrophysical bounds in the parameter space of the mSUGRA scenario $(m$, $M$ ) for $\tan \beta=50, A=0$ and $\mu>0$. The region to the left of the light grey (green) dashed line is excluded by the lower bound on the Higgs mass. The region to the left of the dotted line is excluded by the lower bound on the chargino mass $m_{\tilde{\chi}_{1}^{ \pm}}>103 \mathrm{GeV}$. The region to the right of the black dashed line corresponds to $a_{\mu}^{\mathrm{SUSY}}<7.1 \times 10^{-10}$, and would be excluded by $e^{+} e^{-}$data. The region to the left of the solid line is excluded by $b \rightarrow s \gamma$. The region at the bottom (Stau LSP) is excluded because the lightest stau is the LSP. The region between grey (red) contours fulfils $0.1 \leq \Omega_{\tilde{\chi}_{1}} h^{2} \leq 0.3$, whereas that between black contours indicates the WMAP range $0.094<\Omega_{\tilde{\chi}_{1}^{0}} h^{2}<0.129$. 
0.3. Large gamma-ray fluxes dominated by $b \bar{b}$ final states through $A$-exchange are produced. For slightly smaller values of $M$ one finally arrives to the $A$-pole region where $m_{A} \sim 2 m_{\tilde{\chi}_{1}^{0}}$ and the relic density is too small. Smaller values of $m_{A}$ close this region, producing a decrease in the annihilation cross section, which allows entry into the second corridor with the relic density inside the observational bounds (see e.g. point $\mathbf{C}$ ). For $m_{A}$ too small this second corridor is closed, and the relic density is too large, $\Omega_{\tilde{\chi}_{1}} h^{2}>0.3$.

Finally, there is the narrow Higgsino (focus-point) corridor (see e.g. point D) close to the no electroweak symmetry breaking (nEWSB) region where $\mu^{2}$ becomes negative. In particular, the value of the relic density $\Omega_{\tilde{\chi}_{1}^{0}}$ is affected due to the increase of the Higgsino components of $\tilde{\chi}_{1}^{0}$ with respect to the dominant bino component of most of the parameter space. Thus the relic abundance is placed inside the astrophysical bounds.

The neutralino being mainly Higgsino couples strongly to the $Z$ boson (see Fig. 2) giving rise to a large gamma-ray flux. For these points the neutralino is lighter than in the previous three cases. A full analysis of neutralino annihilation in the universal case parameter space can be found in [54].

Let us finally remark that qualitatively similar allowed corridors are found for the non-universal cases of (3.10). A detailed discussion can be found in Ref. [26].

Concerning the experimental constraints, the lower bounds on the masses of SUSY particles and on the lightest Higgs have been implemented, as well as the experimental bounds on the branching ratio of the $b \rightarrow s \gamma$ process and on $a_{\mu}^{\text {SUSY }}$. We also illustrate these constraints in Fig. 3. Note that we are using $\mu>0$. We will not consider in the calculation the opposite sign of $\mu$ because this would produce a negative contribution for the $g_{\mu}-2$, and, as will be discussed below, we are mainly interested in positive contributions. Recall that the sign of the contribution is basically given by $\mu M_{2}$, and that $M$, and therefore $M_{2}$, can always be made positive after performing an $U(1)_{R}$ rotation.

For $a_{\mu}^{\mathrm{SUSY}}$, we have taken into account the recent experimental result for the muon anomalous magnetic moment [55], as well as the most recent theoretical evaluations of the Standard Model contributions [56]. It is found that when $e^{+} e^{-}$data are used the experimental excess in $\left(g_{\mu}-2\right)$ would constrain a possible SUSY contribution to be $a_{\mu}^{\mathrm{SUSY}}=(27.1 \pm 10) \times 10^{-10}$. At $2 \sigma$ level this implies $7.1 \times 10^{-10} \lesssim a_{\mu}^{\mathrm{SUSY}} \lesssim 47.1 \times 10^{-10}$. It is worth noticing here that when tau data are used a smaller discrepancy with the experimental measurement is found. In order not to exclude the latter possibility we will discuss the relevant value $a_{\mu}^{\text {SUSY }}=7.1 \times 10^{-10}$ throughout the paper. For example, in Fig. 3 the region of the parameter space to the right of this (black dashed) line will be forbidden (allowed) if one consider electron (tau) data.

On the other hand, the measurements of $B \rightarrow X_{s} \gamma$ decays at CLEO [57] and BELLE [58], lead to bounds on the branching ratio $b \rightarrow s \gamma$. In particular we impose on our computation $2.33 \times 10^{-4} \leq B R(b \rightarrow s \gamma) \leq 4.15 \times 10^{-4}$, where the evaluation is carried out using the routine provided by the program micrOMEGAs [59]. This program is also used for our evaluation of $a_{\mu}^{\text {SUSY }}$ and relic neutralino density.

Finally, we have considered in the computation the experimental limit on the $B_{s} \rightarrow$ $\mu^{+} \mu^{-}$branching ratio [60]. Although the upper bound on this process $\mathrm{B}\left(B_{s} \rightarrow \mu^{+} \mu^{-}\right)$ $<2.9 \times 10^{-7}$ does not constrain the parameter space of mSUGRA, it has been stressed 

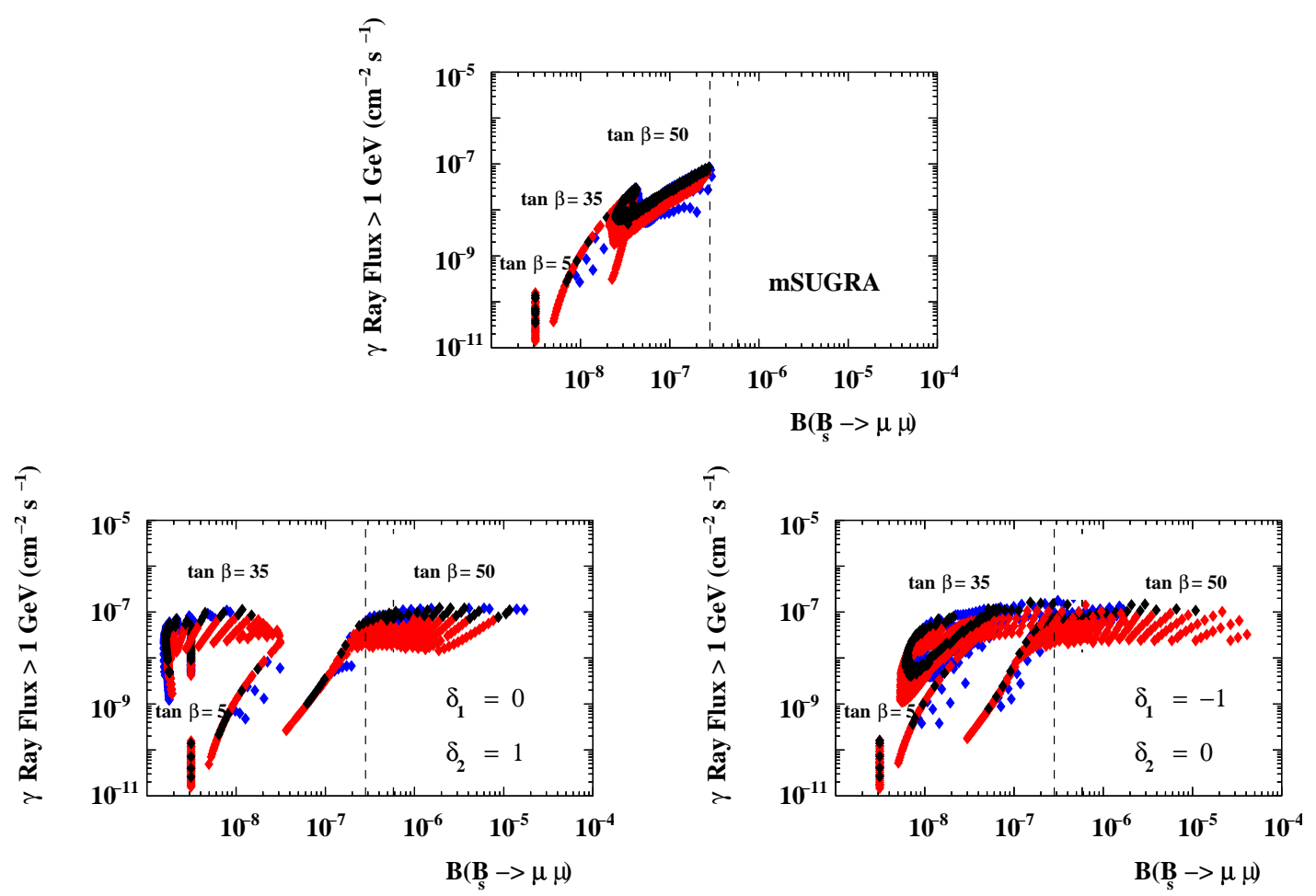

Figure 4: Gamma-ray flux for a threshold of $1 \mathrm{GeV}$ versus $B\left(B_{s} \rightarrow \mu^{+} \mu^{-}\right)$in case $a$ ) mSUGRA of Eq. (3.10) in the text, and non universal Higgs cases b) $\delta_{1}=0, \delta_{2}=1$, and c) $\delta_{1}=-1, \delta_{2}=0$. Points depicted with light grey (red) points have $0.129<\Omega_{\tilde{\chi}_{1}^{0}} h^{2}<0.3$, with black points have $0.094<\Omega_{\tilde{\chi}_{1}^{0}} h^{2}<0.129$, and finally with dark grey (blue) points have $0.03<\Omega_{\tilde{\chi}_{1}^{0}} h^{2}<0.094$ with the appropriate rescaling of the density of neutralinos in the galaxy as discussed in the text. 
recently that in the non-universal cases giving rise to large direct detection cross sections the constraints can be very important $[39,40]$. The reason being that there is a strong correlation between both observables. The branching ratio as well as the cross section increase for low values of the Higgs masses (and large $\tan \beta$ ).

However, in the case of indirect detection of neutralinos through gamma-ray fluxes the correlation is more diluted. In particular, when the neutralino has an important Higgsino component, the $Z$-exchange channel dominates and as a consequence there is no a direct link between the value of the gamma-ray flux and $\mathrm{B}\left(B_{s} \rightarrow \mu^{+} \mu^{-}\right)$. We can see this in Fig. 4, where the gamma-ray flux versus the branching ratio is shown for cases $a$ ), b), c) of (3.10). As mentioned above, for mSUGRA the parameter space is not constrained. Although this is not the case for the non-universal examples, note that still large values of the gamma-ray fluxes can be obtained fulfilling the upper bound for $\mathrm{B}\left(B_{s} \rightarrow \mu^{+} \mu^{-}\right)$.

\section{Comparison with the experiments}

In this section we will compare the theoretical predictions for the indirect detection of neutralinos through gamma-ray fluxes, with the sensitivity of present and future experiments. As discussed in the Introduction, several experiments have already data that apparently cannot be explained with the usual gamma-ray background. Given this situation the comparison between the theoretical computation and the experimental data is crucial. To carry this out we will apply the formulas of Sect. 2.1 to the halo models with baryonic compression discussed in Sect. 2.2, in the context of a general SUGRA theory where non-universal soft terms can be present. We will discuss first the case of space-based detectors such as EGRET and GLAST, and finally atmospheric telescopes such as CANGAROO and HESS.

\section{$4.1 \quad$ EGRET}

The EGRET telescope on board of the Compton Gamma-Ray Observatory has carried out the first all-sky survey in high-energy gamma-rays $(\approx 30 \mathrm{MeV}-30 \mathrm{GeV})$ over a period of 5 years, from April 1991 until September 1996. As a result of this survey, it has detected a signal [31] above about $1 \mathrm{GeV}$, with a value for the flux of about $10^{-8} \mathrm{~cm}^{-2}$ $\mathrm{s}^{-1}$, that apparently cannot be explained with the usual gamma-ray background ${ }^{5}$. The source, possibly diffuse rather than pointlike, is located within the $1.5^{\circ}\left(\Delta \Omega \sim 10^{-3}\right.$ sr) of the galactic center. Due to the lack of precision data in the high energy bins, it seems impossible however to distinguish any annihilation channel leading to this photon excess.

In recent papers $[15,21]$ studies of the perspective of interpreting the spectral features of EGRET data as produced by neutralino annihilation in the galactic center ${ }^{6}$, were carried out. In particular, in Ref. [15] an interesting analysis was carried out in

\footnotetext{
${ }^{5}$ Although alternative explanations for this result have been proposed modifying conveniently the standard theory of galactic gamma ray [61], these turn out to be also controversial [62].

${ }^{6}$ see also [29] for a general analysis.
} 
the context of a toy dark matter model assuming an annihilation cross section $\sigma v \sim$ $3 \times 10^{-26} \mathrm{~cm}^{3} \mathrm{~s}^{-1}$ scaling with the inverse of the relic abundance. Also neutralino dark matter in the mSUGRA context was considered, including experimental constraints coming from accelerator physics. In Ref. [21] the analysis was carried out in the framework of an effective MSSM with non-universal gaugino masses, obtaining that the EGRET excess cannot be reproduced with a NFW profile.

We will show in this section that it is possible to reproduce the spectral features of EGRET data in the framework of general SUGRA, when a NFW profile including adiabatic compression, $\mathrm{NFW}_{c}$, is considered.

The results can be seen in Fig. 5, where several cases of Eq. (3.10) have been studied for a scan on $m$ and $M$ from 0 to $2 \mathrm{TeV}$ and three values of $\tan \beta$ (5, 35 and 50). Only points of the parameter space producing interesting gamma-ray fluxes concerning the EGRET experimental data are shown. Concerning accelerator constraints, these points fulfil the lower bounds on the masses of SUSY particles and Higgs boson, as well as the experimental bounds on the branching ratios of the $b \rightarrow s \gamma$ and $B_{s} \rightarrow$ $\mu^{+} \mu^{-}$processes. They also fulfil WMAP bounds discussed in the previous section. In particular, the thin solid (blue) line corresponds to a $\mathrm{NFW}_{c}$ density profile. Concerning the background of the diffuse gamma-ray flux of the inner galaxy at the energy range of interest for our analysis (from $100 \mathrm{MeV}$ to $10 \mathrm{GeV}$ ), the main production of gammarays is the interaction of cosmic rays (mainly protons and helium nuclei) with the interstellar medium (atomic and molecular hydrogen, and helium). Neutral pions $\pi^{0}$ produced in this process radiate gamma-rays with a spectrum peaked at $\sim 70 \mathrm{MeV}$ $\left(m_{\pi} / 2\right)$, dropping at high energies with an energy power law which follows the initial cosmic ray spectrum of the form $E^{-\alpha}$ with $\alpha \sim 2.7[15,62]$. The normalization factor has been fixed to have the best fit in agreement to the standard scenario, $\phi_{b}=$ $2 \times 10^{-6} \mathrm{~cm}^{-2} \mathrm{~s}^{-1} \mathrm{GeV}^{-1} \mathrm{sr}^{-1}$ for $2 \mathrm{GeV}<E_{\text {bin }}<4 \mathrm{GeV}[15]$.

As one can see, with small values of $\tan \beta$ we are not able to find points reproducing EGRET data. However, interesting points can be found for larger values of $\tan \beta$. In particular, we show in the figure the cases $\tan \beta=35,50$ and one can see that with non-universal cases EGRET data can be reproduced. This is obtained for neutralino masses between 150 and $600 \mathrm{GeV}$.

Let us remark that it is possible to differentiate each point of the parameter space $(m, M)$ by its gamma-ray spectrum. Indeed, just a look at Fig. $5 \mathrm{~b}$ for $\tan \beta=35$ shows two kind of spectra. The two highest lines correspond to points with $(m, M)=(533$ $\mathrm{GeV}, 400 \mathrm{GeV})$ and $(733 \mathrm{GeV}, 533 \mathrm{GeV})$, and they are located inside the Higgsino region dominated by $f \bar{f}$ final states through $Z$-exchange (see e.g. point $\mathbf{D}$ of Fig. 3). On the other hand, the lower line corresponds to $(m, M)=(266 \mathrm{GeV}, 666 \mathrm{GeV})$, which lies inside the stau coannihilation region (see e.g. point $\mathbf{A}$ of Fig. 3), where the annihilation is weak but dominated by $A$-exchange, and the spectrum is thus a $b \bar{b}$ one. In the case of Fig. $5 \mathrm{c}$ with $\tan \beta=35$, the higher fluxes corresponds to the closing of the $A$-pole (see e.g. point $\mathbf{C}$ of Fig. 3 ) whereas the lower flux spectrum is obtained through the opening of the $A$-pole (see e.g. point $\mathbf{B}$ of Fig. 3 ). The same remarks can be made for the other cases illustrated in Fig. 5.

We have also carried out the same analysis for a Moore et al. profile including adiabatic compression, Moore $_{c}$, as shown in the figure with a dashed line for the differ- 

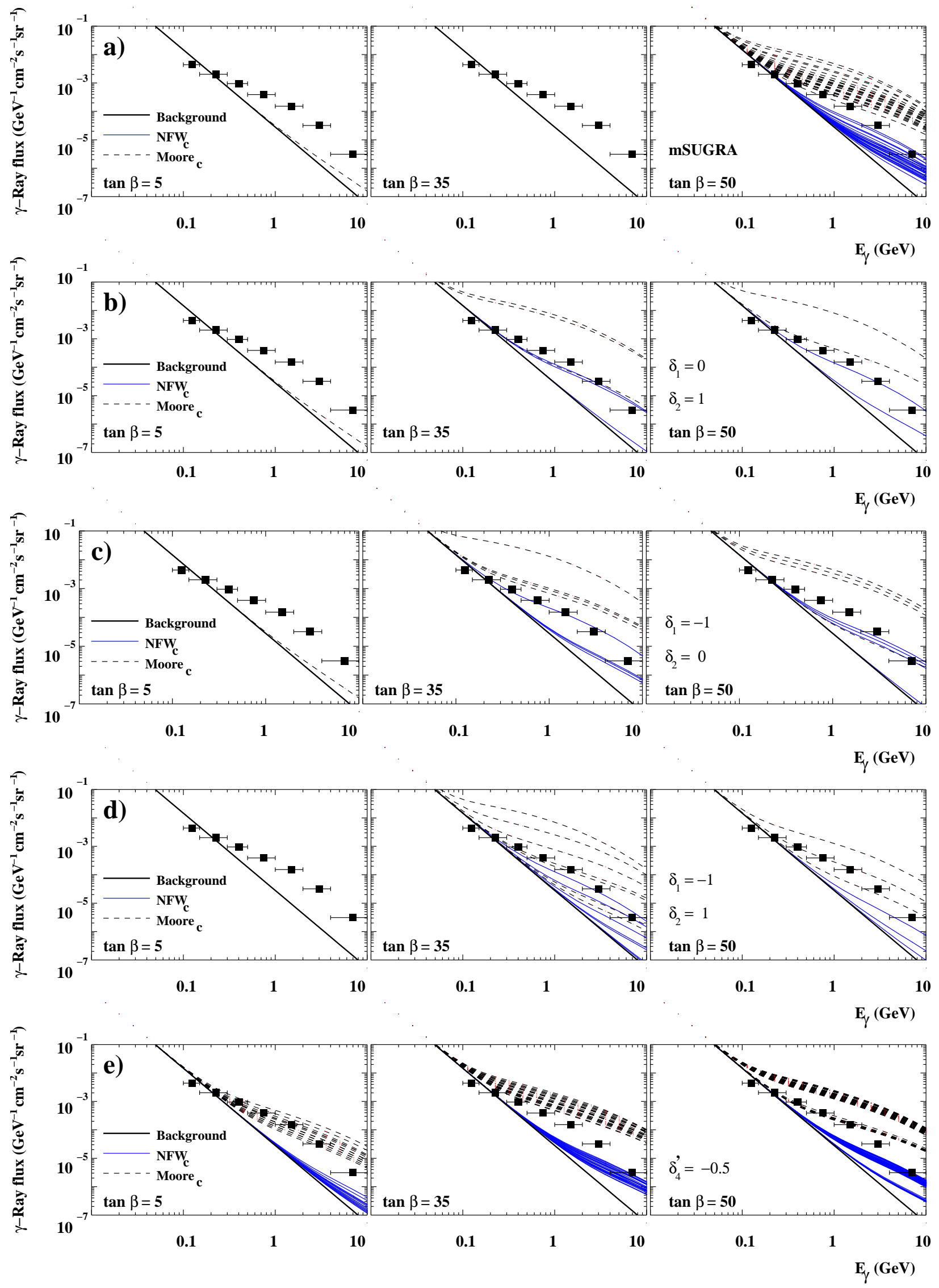

Figure 5: Gamma-ray spectra $\Phi_{\gamma}\left(E_{\gamma}\right)$ from the galactic center as functions of the photon energy for the SUGRA cases discussed in Eq. (3.10) for $\tan \beta=5,35,50, A=0$ and $\mu>0$, compared with data from the EGRET experiment. NFW and Moore et al. profiles with adiabatic compression are used with $\Delta \Omega \sim 10^{-3}$ sr. All points shown after a scan on $m$ and $M(0-2000 \mathrm{GeV})$ fulfil the accelerator constraints discussed in the text, and WMAP bounds. 
ent points of the parameter space. Clearly, due to the very singular behaviour of this profile many points are constrained by EGRET data.

\subsection{GLAST}

Crucial information on the origin and nature of the EGRET excess at the galactic center will be provided by another space-based detector, GLAST. This is scheduled for launch in 2007, and will perform an all-sky survey covering a larger energy range $(\approx 10-300 \mathrm{GeV})$ than EGRET, with a wider effective area and a better resolution in energy. The experiment will also allow to search for an angular distribution of the dark matter halo. Indeed, with an angular resolution of $0.1^{\circ}\left(\Delta \Omega \sim 10^{-5} \mathrm{sr}\right)$, GLAST will be able to point and analyse the inner center of the Milky Way $(\sim 7 \mathrm{pc})$. Even if alternative explanations solve the data problem pointed out by EGRET, GLAST will be able to detect a much smaller flux of gamma rays from dark matter $\sim 10^{-11} \mathrm{~cm}^{-2}$ $\mathrm{s}^{-1}$.

We summarize the results of our analysis in Fig. 6, using the same parameter space as in Fig. 5, and a $\mathrm{NFW}_{c}$ profile. There, the values of the total gamma-ray flux $\Phi_{\gamma}$ for a threshold of $1 \mathrm{GeV}$ allowed by all experimental constraints as a function of the neutralino mass $m_{\tilde{\chi}_{1}^{0}}$ are depicted. Concerning the predicted sensitivity for the experiment, we follow Ref. [65] and show on the plot the $5 \sigma$ sensitivity curves assuming an angular resolution of $0.1^{o}\left(\Delta \Omega=10^{-5}\right)$, and a total effective pointing time of 30 days for the satellite. We observe that, basically, the whole parameter space of general SUGRA will be tested by GLAST. Note that even for the mSUGRA case points corresponding to a value of $\tan \beta$ as small as 5 will be reached by GLAST. This is a remarkable result if we realise that interesting direct detection experiments, such as e.g. EDELWEIS-II or CDMS-II, will only be able to cover small regions of the parameter space. More poweful detectors such as GENIUS will cover larger regions, but still not too large compared with the parameter space of general SUGRA. In contrast, we have observed that indirect detection experiments will be able to test the parameter space of SUGRA ${ }^{7}$.

It is also worth noticing that many points are already constrained by EGRET data. See e.g. case $c$ ) with $\tan \beta=35$. Let us remark that in Fig. 6 EGRET line corresponds to $\Delta \Omega \sim 10^{-3} \mathrm{sr}$, and therefore for its study all points in the figure should be rescaled

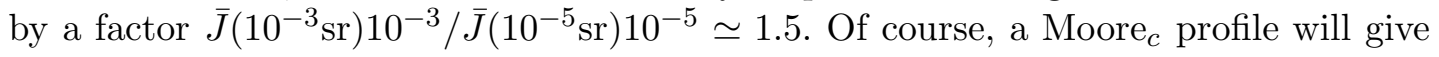
rise to larger fluxes.

\subsection{CANGAROO}

Recently, the CANGAROO-II atmospheric Cherenkov telescope has made a significant detection of gamma rays from the galactic center region [32]. In particular, the collaboration has published the spectrum obtained in six energy bins, from 200 $\mathrm{GeV}$ to $3 \mathrm{TeV}$. Observations taken during 2001 and 2002 have detected a statistically significant excess at energies greater than $\sim 250 \mathrm{GeV}$, with an integrated flux

\footnotetext{
${ }^{7}$ See also Ref. [28] for a comparison between direct and indirect dark matter search without using compressed halo models.
} 

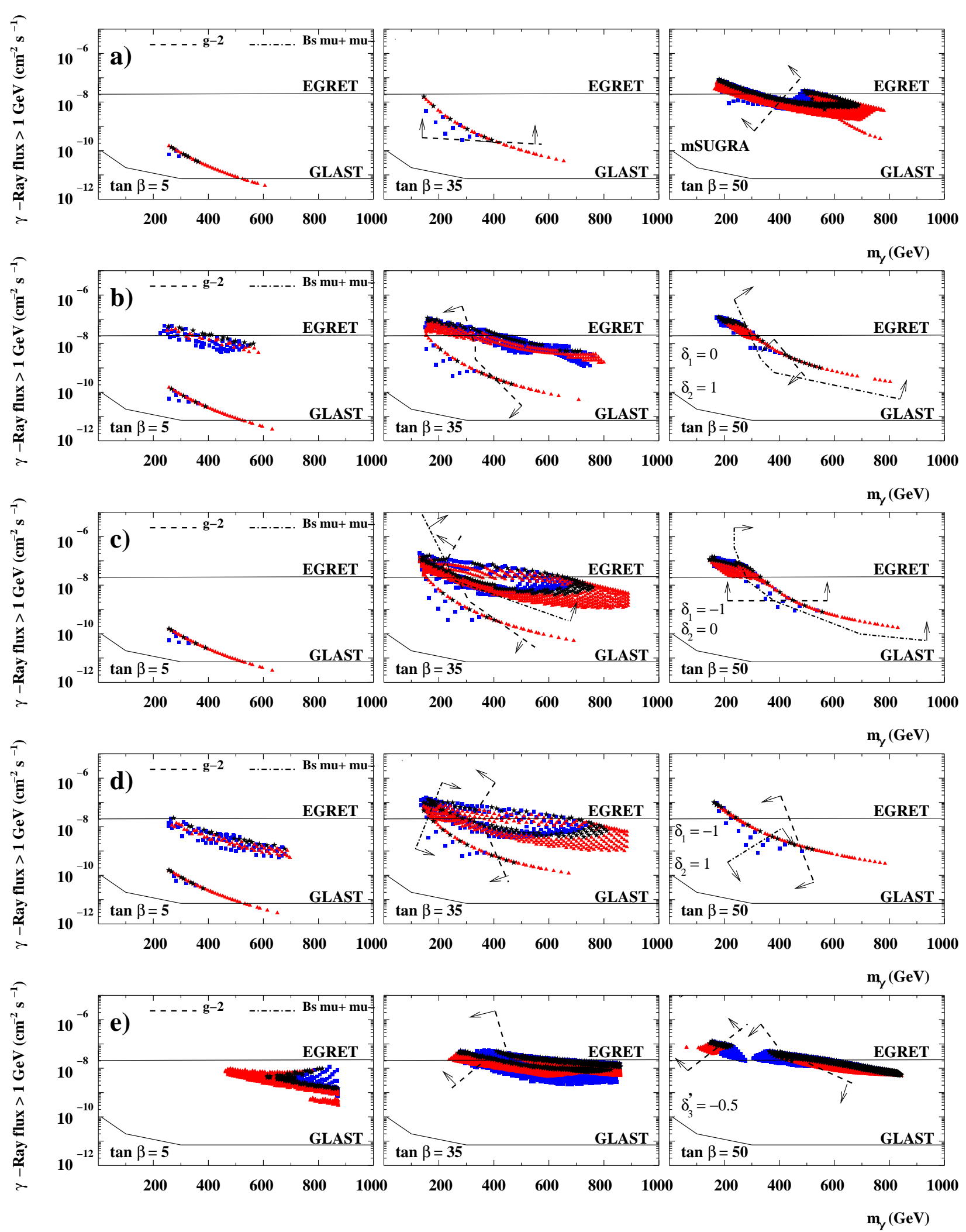

Figure 6: Scatter plot of the gamma-ray flux $\Phi_{\gamma}$ for a threshold of $1 \mathrm{GeV}$ as a function of the neutralino mass $m_{\chi}$ for the SUGRA cases discussed in Eq. (3.10), and for the same parameter space used in Fig. 5. A NFW profile with adiabatic compression is used with $\Delta \Omega \sim 10^{-5} \mathrm{sr}$. All points in the figure fulfil the $b \rightarrow s \gamma$ bounds. For $\tan \beta=5$ all points have $a_{\mu}^{\mathrm{SUSY}}<7.1 \times 10^{-10}$. For the other values of $\tan \beta, a_{\mu}^{\mathrm{SUSY}}>7.1 \times 10^{-10}$ is fulfilled for the points located on the arrow side of the dashed lines, and $\mathrm{B}\left(B_{s} \rightarrow \mu^{+} \mu^{-}\right)<2.9 \times 10^{-10}$ for those on the arrow side of the dot-dashed lines. Points depicted with light grey (magenta) triangles have $0.129<\Omega_{\tilde{\chi}_{1}^{0}} h^{2}<0.3$, with black stars have $0.094<\Omega_{\tilde{\chi}_{1}^{0}} h^{2}<0.129$, and finally with dark grey (blue) boxes have $0.03<\Omega_{\tilde{\chi}_{1}^{0}} h^{2}<0.094$ with the appropriate rescaling of the density of neutralinos in the galaxy as discussed in the text. Solid lines denote the $5 \sigma$ sensitivity curves for satellites. From top to bottom, the first solid line corresponds to the signal reported by EGRET, and the upper area bounded by the second solid line will be analyzed by GLAST experiment. 

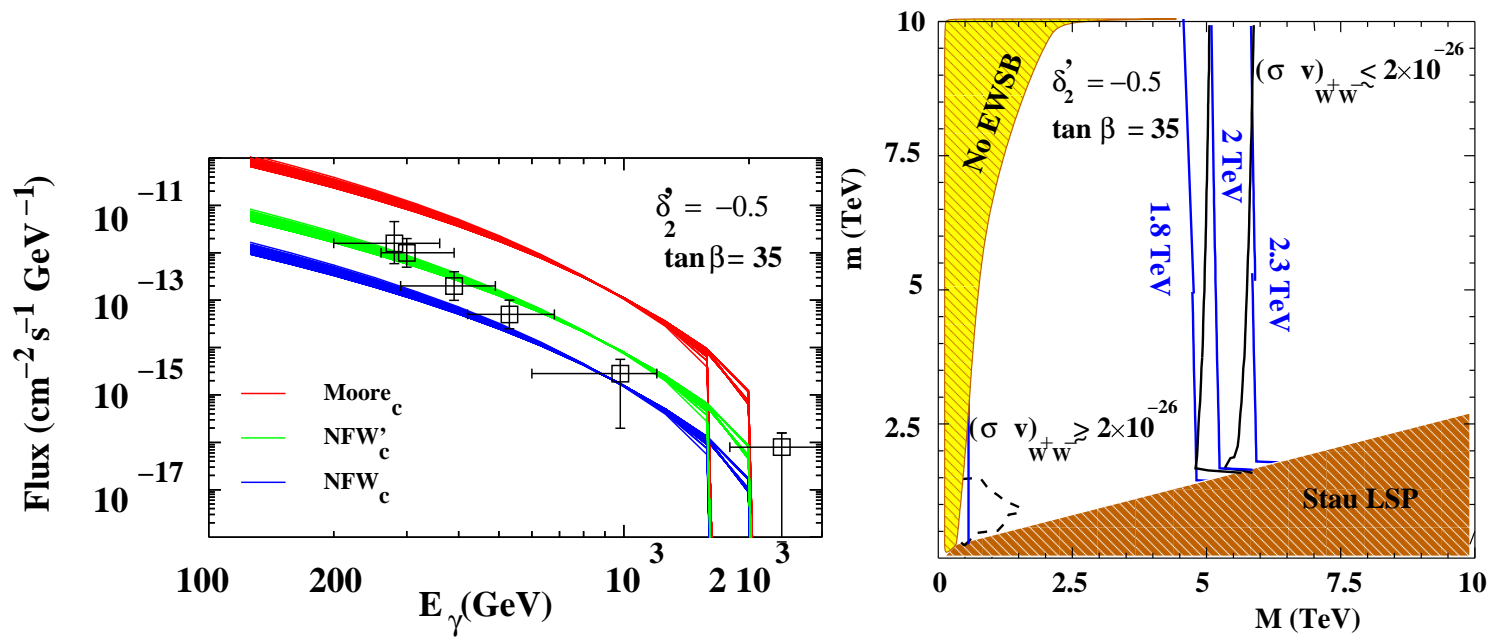

Figure 7: Left frame: Gamma-ray spectra from the galactic center as functions of the photon energy for the case $f$ ) discussed in Eq. (3.10) with non-universal gaugino masses $M_{2}=0.5 M$, for $\tan \beta=35, A=0$ and $\mu>0$, compared with data from the CANGAROO-II experiment. NFW and Moore et al. profiles, as well as the average profile defined in Sect. 2.2 with adiabatic compression are used with $\Delta \Omega \sim 10^{-5}$ sr. All points shown after a scan on $m$ and $M(0-10 \mathrm{TeV})$ fulfil the accelerator constraints discussed in the text, and WMAP bounds. Right frame: Experimental and astrophysical bounds in the parameter space $(m, M)$. The conventions and constraints are the same as in Fig. 3. Blue lines are contours of neutralino masses.

of $\sim 2 \times 10^{-10}$ photons $\mathrm{cm}^{-2} \mathrm{~s}^{-1}$. These measurements indicate a very soft spectrum $\propto E^{-4.6 \pm 0.5}$. As discussed in Ref. [63], this signal could be fitted with a spectrum of a $\mathrm{TeV}$-dark matter candidate. It is interesting to see whether it is possible to obtain such a candidate in SUGRA scenarios imposing the accelerator and WMAP constraints, and withing the framework of adiabatically compressed halos.

As we can see in Fig. 3, most of the regions of the parameter space of mSUGRA which satisfy WMAP constraints give mainly a sub-TeV spectrum in scalar and gaugino sectors. It is thus very difficult to find a dark matter candidate fitting the observations made by the CANGAROO-II experiment. Nevertheless, it is possible to extend the allowed region if we release the gaugino universality. One way to achieve this is to decrease $M_{2}$ while keeping $M_{1}=M_{3}$ at $M_{G U T}$. This allows to reduce significantly the relic abundance of the wino-like neutralino, through its coannihilation with the lightest chargino $\chi_{1}^{+}$. Such an scenario, well motivated e.g. in anomaly mediation models, predicts higher values for the gaugino masses fulfilling WMAP constraints than those of universal cases, typically around the $\mathrm{TeV}$ scale, which is precisely the region favoured by CANGAROO-II results.

As an illustration, let us consider the case $f$ ) in Eq. (3.10) giving rise to $M_{2}=0.5 \mathrm{M}$. We show this case in Fig. 7. There all points fulfil the lower bounds on the masses of SUSY particles and Higgs boson, as well as the experimental bounds on the branching 

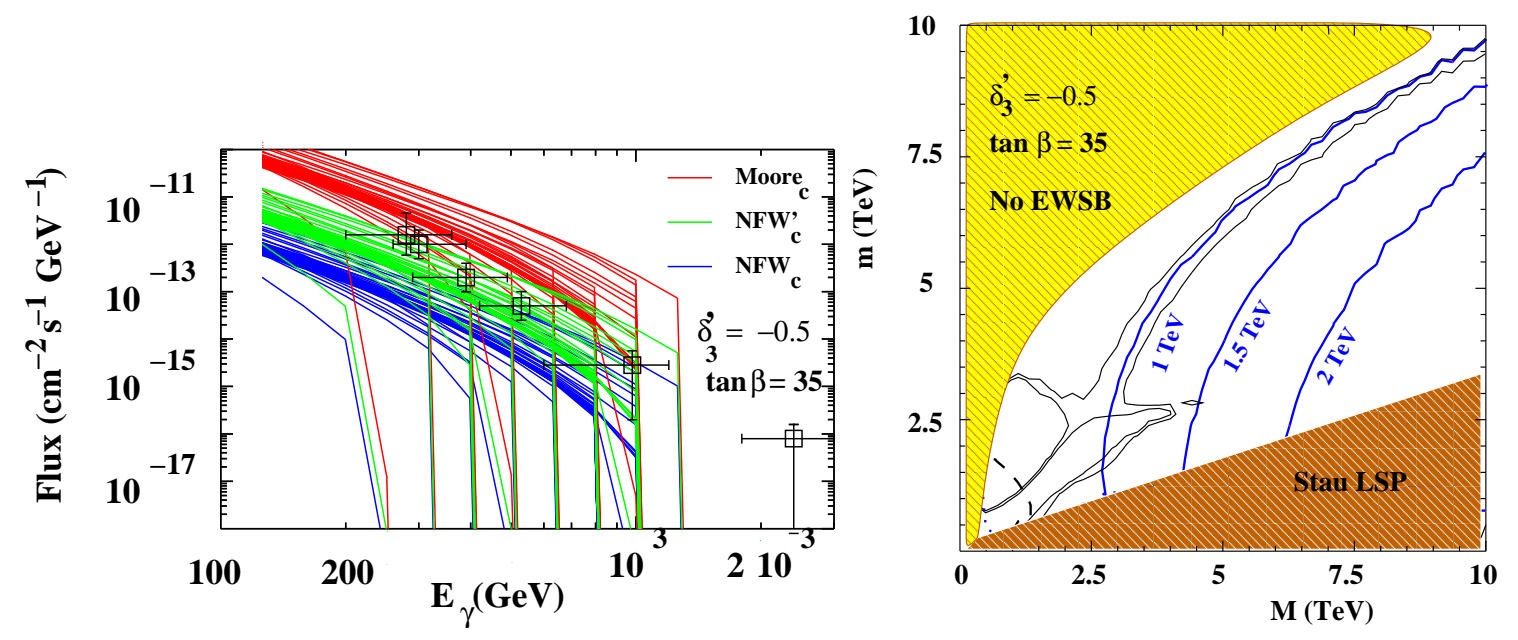

Figure 8: The same as in Fig. 7 but for the non-universal case e) with $M_{3}=0.5 M$.

ratios of the $b \rightarrow s \gamma$ and $B_{s} \rightarrow \mu^{+} \mu^{-}$processes. They also fulfil the WMAP bounds. As we can see in the left and right frames of the figure, there is an interesting region of the parameter space fulfilling the WMAP constraints and giving rise to a sufficiently large flux to fit the data from CANGAROO-II. Let us remark that the highest energy bin shown (of about $2.5 \mathrm{TeV}$ ) is less than $1 \sigma$ in excess of a null result, so it should be considered only as an upper limit. Excluding this point from the analysis, we see that this non-universal model can fit CANGAROO data and WMAP bounds in halos models with profiles between $\mathrm{NFW}_{c}$ and Moore $_{c}$. In particular, this is the case of the scenario discussed in Sect. 2.2, $\mathrm{NFW}_{\mathrm{c}}^{\prime}$, with a value of $\bar{J}$ given by $5 \times \bar{J}_{\mathrm{NFW}_{\mathrm{c}}}$, as can be seen in the left frame of the figure.

It is worth noticing that the parameter space where WMAP constraints are fulfilled corresponds to a narrow range of the neutralino mass $(\sim 2 \mathrm{TeV})$, as can be seen in the right frame of Fig. 7. This region is mainly independent of $\tan \beta$ and the value of $\delta_{2}^{\prime}$ as far as the neutralino is wino like $\left(\delta_{2}^{\prime}<-0.45\right)$. In this region of the parameter space, the relic density calculation is governed by the $\chi_{1}^{0}-\chi_{1}^{+}$coannihilation into $W^{+} W^{-}$ $\left(m_{\chi_{1}^{0}} \sim m_{\chi_{1}^{+}} \sim M_{2}\right)$, whereas the flux is largely dominated by the annihilation process $\chi_{1}^{0} \chi_{1}^{0} \rightarrow W^{+} W^{-}$through $t$-chanel $\chi_{1}^{+}$exchange $\left(\left.\sigma v\right|_{W W} \sim 10^{-26} \mathrm{~cm}^{2} \mathrm{~s}^{-1}\right)$.

Another possibility is to produce a neutralino-LSP with a higher higgsino component. Indeed, as discussed in Ref. [26], decreasing the value of $M_{3}$ while keeping $M_{2}=M_{1}$ at $M_{G U T}$ increases the Higgsino fraction of the neutralino through the renormalization group equations (RGEs), and, as a consequence, its coupling to the $Z$ boson. In this case, the relic density constraint can be satisfied more easily because the contribution from the annihilation into gauge bosons channel is more important and the focus point region is much wider. Another consequence of the decreasing of $M_{3}$ at $M_{G U T}$ is the decreasing of the pseudoscalar mass $m_{A}$ through $m_{H_{2}}$. In the left frame of Fig. 8 we show the case $e$ ) of Eq. (3.10) with $M_{3}=0.5 M$. For $\tan \beta$ larger than about 


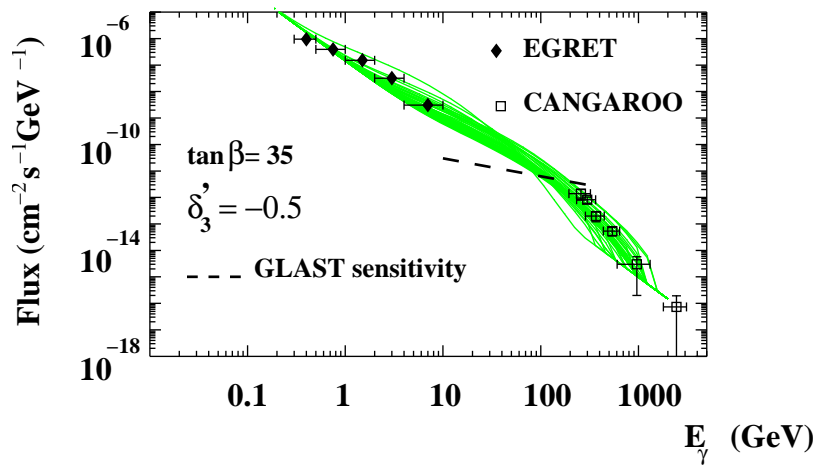

Figure 9: The same as in the left frame of Fig. 7 but for the non-universal case e) with $M_{3}=0.5 M$, compared with data for EGRET and CANGAROO-II experiments and the expected GLAST sensitivity. Here only the average profile defined in Sect. 2.2 with adiabatic compression, $\mathrm{NFW}_{\mathrm{c}}^{\prime}$, is used.

10, the neutralino annihilation into fermions (mainly $b \bar{b}$ ) starts to dominate. Eventually, the focus point and Higgs annihilation regions merge, for example for $\tan \beta=35$ and $M \approx 2 \mathrm{TeV}$, as was already pointed out by the authors of Ref. [64]. This region is clearly visible in the right frame of Fig. 8. Whereas in the previous analised case $f$ ) the WMAP constraint was fulfilled through one main chanel ( $\chi_{1}^{0} \chi_{1}^{+}$coannihilation), the non-universality of $M_{3}$ offers more possibilities through the exchange of Higgses. That explains why the region allowed by WMAP constraint is broader in the right frame of Fig. 8 than in Fig. 7.

Of course in these models the SUSY spectrum is very heavy. For example, for a point with $m=5 \mathrm{TeV}, M=5 \mathrm{TeV}$ reproducing CANGAROO-II data, the spectrum in case $e$ ) $(f)$ consists of heavy squarks, $m_{\tilde{q}} \sim 8 \mathrm{TeV}(10 \mathrm{TeV})$, neutralinos and charginos $m_{\chi_{1}^{0}} \sim m_{\chi_{1}^{+}} \sim 1 \mathrm{TeV}(2 \mathrm{TeV})$, and also heavy higgses $m_{A} \sim 5 \mathrm{TeV}(6 \mathrm{TeV})$. If we do not take into account the potential fine-tuning problem associated with such models, and consider them reliable, this would be a difficult situation for any hope of discovering SUSY at colliders, since the only detectable particle would be the lightest Higgs. We have plotted for information the $2 \sigma$ standard deviation $a_{\mu}^{\text {SUSY }}=7.1 \times 10^{-10}$ with dashed line in the right frames of Figs. 7 and 8. Only an unambiguous evidence for a non-zero contribution to $\delta a_{\mu}$ would restrict this class of models with heavy sparticles.

Let us finally mention that we have also try to reproduce the flux measured by CANGAROO-II using cases $b$ ), c) and $d$ ) with non universal scalars. They give rise to a neutralino too light to be able to reproduce those data.

\subsection{Combining EGRET and CANGAROO}

After analysing EGRET and CANGAROO-II data, it seems natural to try to fit both experiments with only one non-universal scenario. We notice here that a similar ex- 
ercise has been carried out by the authors of Ref. [65]. The baryonic cooling effect on the fluxes gives us the order of magnitude needed to fit with both data with a $1 \mathrm{TeV}$ neutralino in the non-universal case $e$ ) with $M_{3}=0.5 M$. It is worth noticing that the CANGAROO-II collaboration in [32] pointed out already that the EGRET and CANGAROO-II data can be relatively smoothly connected with a cutoff energy of 1-3 $\mathrm{TeV}$. In our case, for that we need to use the $\mathrm{NFW}_{c}{ }_{c}$ scenario discussed in Sect. 2.2. Typical points of the parameter space fullfilling all experimental constraints and fitting both set of data lie between $(m=800 \mathrm{GeV}, M=800 \mathrm{GeV})$ and $(m=3 \mathrm{TeV}, M=3$ $\mathrm{TeV})$.

It is also interesting to see the complementarity of GLAST with EGRET and CANGAROO. GLAST will perform an all-sky survey detection of fluxes with energy from $1 \mathrm{GeV}$ to $300 \mathrm{GeV}$, exactly filling the actual lack of experimental data in this energy range (see Fig. 9), and checking the CANGAROO results. Indeed, we have calculated that the integrated gamma ray flux for such a signal will be around $5 \times 10^{-11} \mathrm{~cm}^{-2} \mathrm{~s}^{-1}$. We have shown this sensitivity curve in Fig. 9 for $\Delta \Omega=10^{-5}$, which is the typical detector acceptance, following the prescriptions given in [65]. We clearly see that GLAST will help to cover the entire spectrum.

We have also tried to reproduce the observed data using a wino-like neutralino arising from the non-universal case $f$ ) with $M_{2}=0.5 M$. But any model of this type fulfilling WMAP bounds gives rise to a too heavy neutralino-LSP $(\sim 2 \mathrm{TeV})$ whose spectral features cannot explain at the same time both the CANGAROO-II and EGRET excess.

\subsection{HESS}

The HESS Cherenkov telescope experiment has recently published new data on gamma rays, detecting a signal from the galactic center [33]. The measured flux and spectrum differ substantially from previous results, in particular those reported by the CANGAROO collaboration, exhibiting a much harder power-law energy spectrum with spectral index of about -2.2 and extended up to $9 \mathrm{TeV}$. The authors of [33] already pointed out that if we assume that the observed gamma rays represent a continuum annihilation spectrum, we expect $m_{\chi} \gtrsim 12 \mathrm{TeV}$. Actually such a heavy neutralino-LSP is not natural in the framework of a consistent supergravity model when we impose the renormalisation group equations and radiative electroweak symmetry breaking.

Although we performed some scans in all non universality directions using numerical dichotomy methods, no point in the parameter space in any non-universal case studied was able to give a several $10 \mathrm{TeV}$ neutralino satisfying WMAP constraint but this can be sensitive to the RGE and relic density calculation codes.

Nevertheless, without RGE and taking soft parameters at the electroweak scale, the constraints are easier to evade. On top of that, in a very effective approach using completely free parameters and couplings in cross sections, neutralinos with $m_{\chi} \gtrsim$ $10 \mathrm{TeV}$ and $\Omega_{\chi} h^{2} \sim W M A P$ may certainly be fine tuned. We did not adopt such approaches since the MSSM is motivated by high energy and theoretical considerations. In this section, we analyze in a quite model-independant way the conditions required on the particle physics field to fit with the HESS data thanks to dark matter annihilation. 
a)
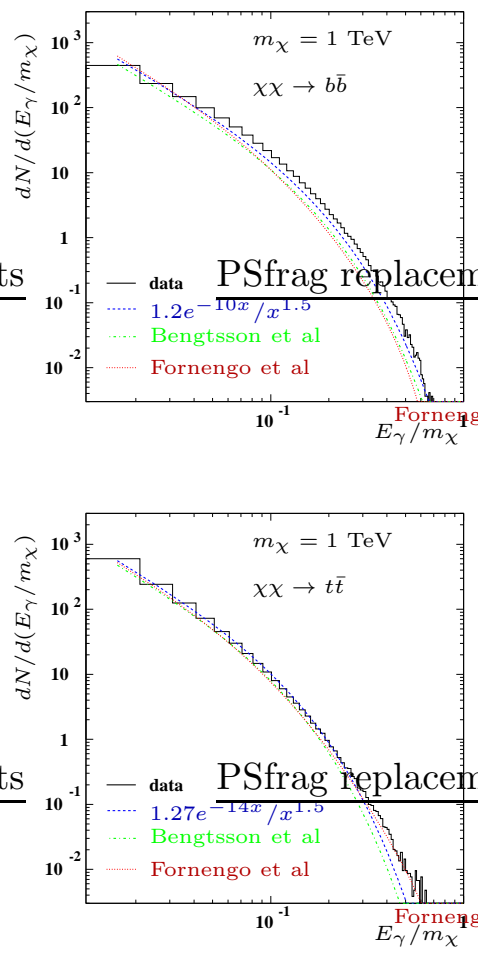

c)

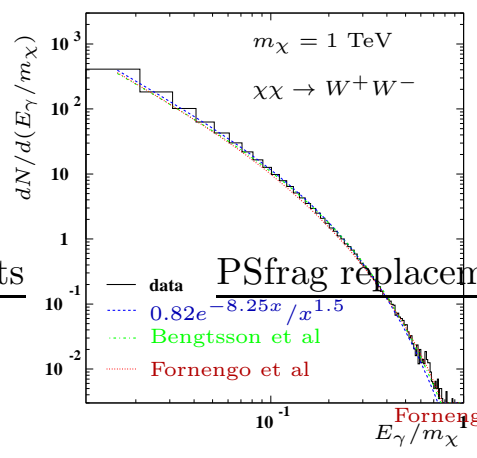

d)

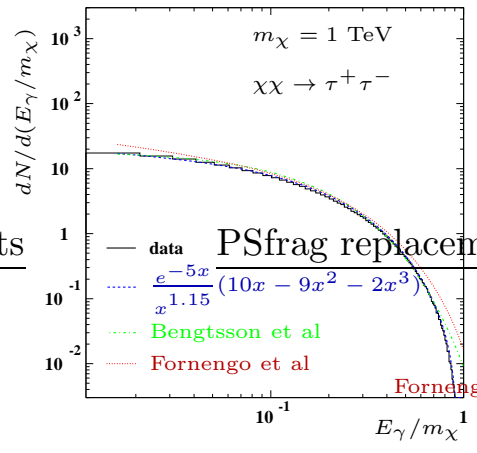

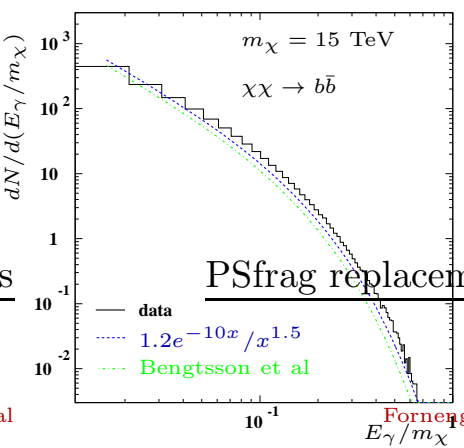
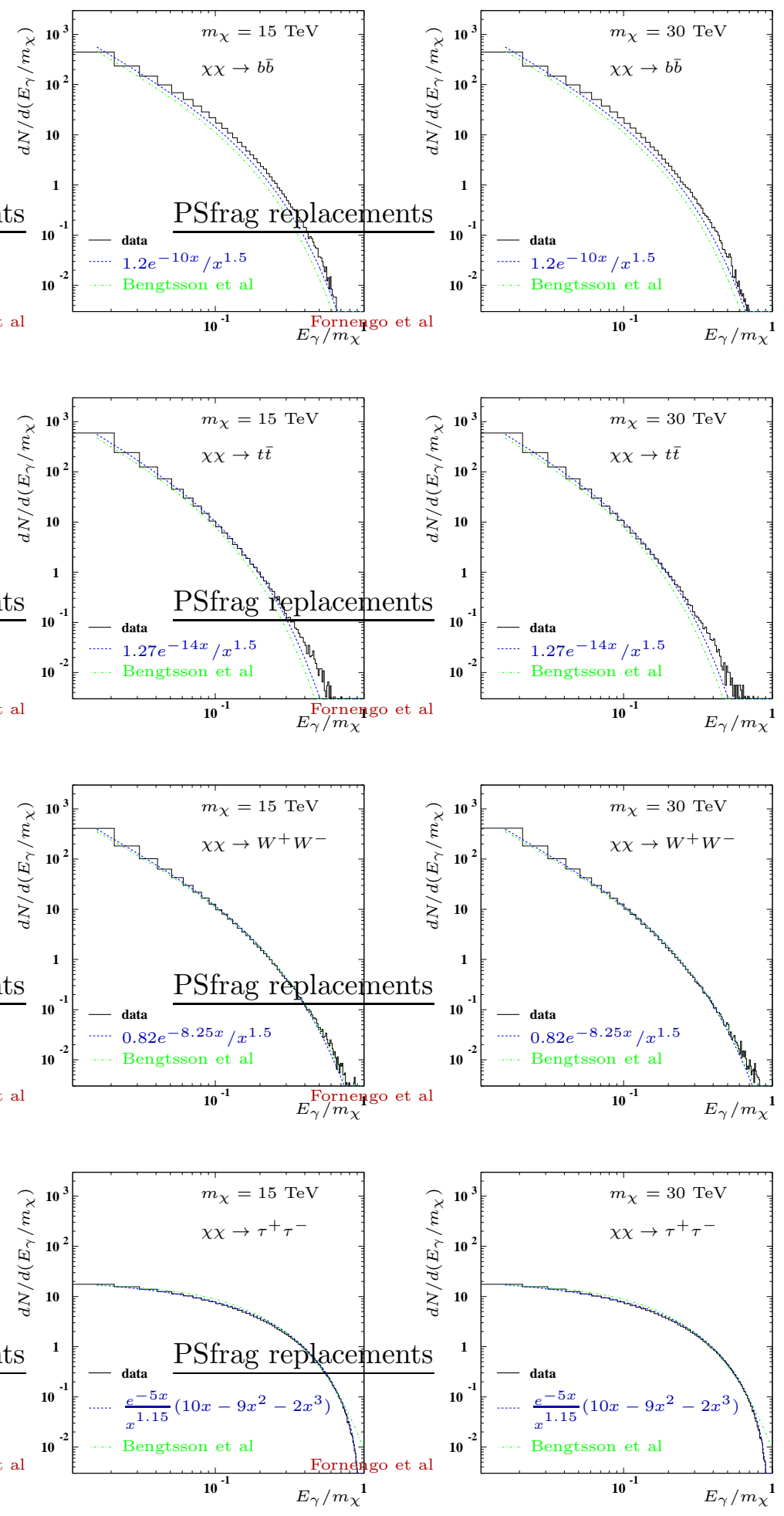

Figure 10: PYTHIA simulations for gamma spectrum coming from the decays of dark matter particle annihilation products ( a) $b \bar{b}$, b) $t \bar{t}$, c) $W^{+} W^{-}$, d) $\tau^{+} \tau^{-}$) for three dark matter particle masses: 1,15 and $30 \mathrm{TeV}$. We show the data, our fit results and also compare with previous studies $[13,65]$. 
We performed a simulation with the PYTHIA ${ }^{8}$ package [66] to evaluate the gammaray fluxes from hadronisation and radiative processes. We consider a dark matter particle annihilating at rest in the $b \bar{b}, t \bar{t}, W^{+} W^{-}$and $\tau^{+} \tau^{-}$final states. We first require a $1 \mathrm{TeV}$ mass (i.e. $E_{c m}=2 \mathrm{TeV}$ ) to check our results with previous studies $[13,65]$ and then generate two relevant masses for HESS data: $15 \mathrm{TeV}$ and $30 \mathrm{TeV}$ (i.e. respectively $E_{c m}=30$ and $60 \mathrm{TeV}$ ). The results are shown on figure 10. Even if more precise fits can be obtained with more parameters, the annihilation spectra can be quite simply approximated by :

$$
\frac{d N_{\gamma}^{i}}{d x}=\frac{a_{i} e^{-b_{i} x}}{x^{1.5}}
$$

where $x=E_{\gamma} / m_{\chi}$ and we obtained $(a, b)=(1.2,10$. $)$ for $b \bar{b}^{9},(a, b)=(1.27,14)$ for $t \bar{t}$ and $(a, b)=(0.82,8.25)$ for $W^{+} W^{-}$. For $\tau^{+} \tau^{-}$we used the function proposed in [65]

$$
\frac{d N_{\gamma}^{i}}{d x}=x^{a_{\tau}}\left(b_{\tau} x+c_{\tau} x^{2}+d_{\tau} x^{3}\right) e^{-e_{\tau} x}
$$

to well reproduce the harder $\tau^{+} \tau^{-}$spectrum and found $\left(a_{\tau}, b_{\tau}, c_{\tau}, d_{\tau}, e_{\tau}\right)=(-1.15,10,-9 .-$ $2,5)$.

The gamma ray flux was expressed in Eq. (2.5), with $\left\langle\sigma_{i} v\right\rangle=B R_{i}\langle\sigma v\rangle, B R_{i}$ being the branching ratio of annihilation channel $i$.

With this parameterization, the gamma flux can be rewritten as

$$
\frac{d \Phi_{\gamma}}{d E_{\gamma}}\left(\mathrm{cm}^{-2} \mathrm{~s}^{-1} \mathrm{GeV}^{-1}\right)=\frac{0.94 \times 10^{-13}}{m_{\chi}(\mathrm{GeV})} \sum_{i} \frac{d N_{\gamma}^{i}}{d x} B R_{i}\left(\frac{\langle\sigma v\rangle}{10^{-29} \mathrm{~cm}^{3} \mathrm{~s}^{-1}}\right)\left(\frac{100 \mathrm{GeV}}{m_{\chi}}\right)^{2} \bar{J}(\Delta \Omega) \Delta \Omega
$$

where $m_{\chi},\langle\sigma v\rangle, B R_{i}$ are now considered as free parameters and the astrophysics contribution $\bar{J}$ is fixed by our halo density profiles. The results are shown in Fig. 11.

We point the fact that we do not only compare the spectrum shape of the signal with possible dark matter annihilation explanation. Indeed, it should be noticed that our compressed halo profiles give rise to absolute gamma fluxes within the HESS data order of magnitude with $\langle\sigma v\rangle$ values in agreement with the WMAP requirement.

According to our analysis, interpreting the HESS data in terms of dark matter particle annihilation is possible for a mass in the range $10 \mathrm{TeV} \lesssim m_{\chi} \lesssim 30 \mathrm{TeV}$ and annihilation should be dominated by $b \bar{b}, t \bar{t}$ or $W^{+} W^{-}$final states with a possible $\tau^{+} \tau^{-}$contribution ${ }^{10}$. The values for $\langle\sigma v\rangle$ required for NFW compressed and Moore compressed are respectively around $10^{-25} \mathrm{~cm}^{3} \mathrm{~s}^{-1}$ and $10^{-27} \mathrm{~cm}^{3} \mathrm{~s}^{-1}$.

We also tried to see if it was possible to explain at the same time both HESS and EGRET excess by invoking a very heavy neutralino. We can easily predict the differential flux around $1 \mathrm{GeV}$ for a $15 \mathrm{TeV}$ neutralino. Using Eq. (4.11) for a $t \bar{t}$ distribution (the one producing the less energetic photons) normalised with the HESS data

\footnotetext{
${ }^{8}$ We used january 2005 release of PYTHIA (version 6.317).

${ }^{9}$ Despite the Hill function, the $b \bar{b}$ case can suggest to relaxe the -1.5 exponent to get better fit.

${ }^{10}$ In the MSSM, $\frac{B R_{\tau}+\tau_{-}-}{B R_{b \bar{b}}} \sim \frac{1}{3} \frac{m_{\tau}^{2}}{m_{b}^{2}} \sim 0.15$ for pseudo scalar exchange.
} 


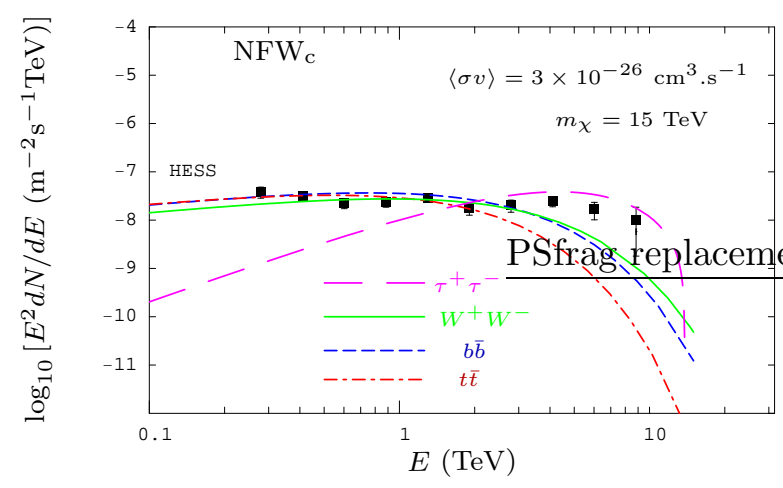

a)

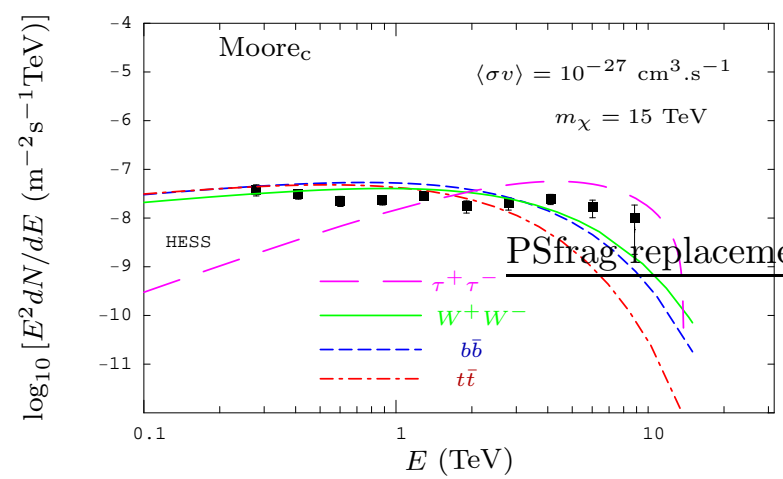

c)

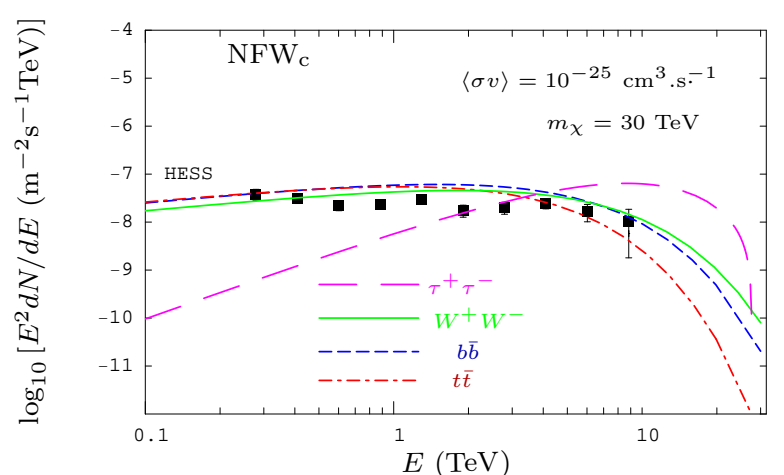

b)

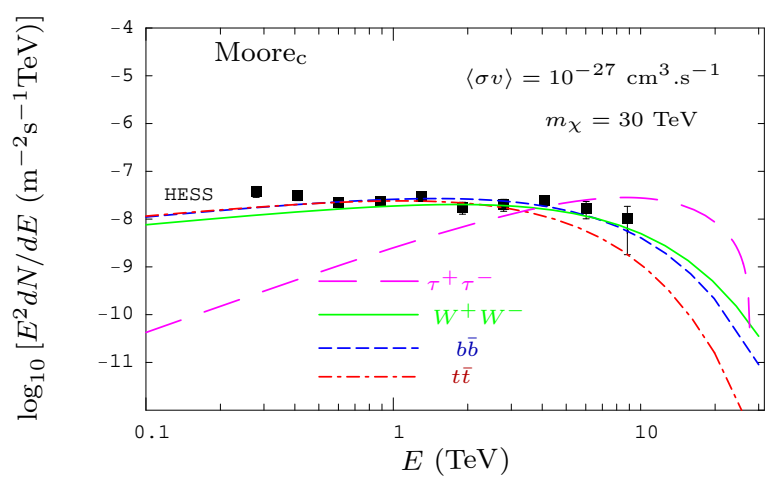

d)

Figure 11: Dark matter annihilation versus HESS data for : NFW compressed in a) and b), Moore et al. compressed in c) and d). 
in the $2.6 \mathrm{TeV}$ energy bin $\left(2 . \times 10^{-16} \mathrm{~cm}^{-2} \mathrm{~s}^{-1} \mathrm{GeV}^{-1}\right)$ we obtain : $d N_{\gamma}^{t} / d E_{\gamma}(1 \mathrm{GeV}) \sim$ $7.1 \times 10^{-12} \mathrm{~cm}^{-2} \mathrm{~s}^{-1} \mathrm{GeV}^{-1}$, which is far below the EGRET excess data in the $1 \mathrm{GeV}$ energy bin $\left(1.52 \times 10^{-7} \mathrm{~cm}^{-2} \mathrm{~s}^{-1} \mathrm{GeV}^{-1}\right)$. At the same time, we have numerically checked that such a heavy neutralino cannot be invoked to explain the EGRET excess in any of its energy bin. During this work, we saw that in a recent note added in [65], the authors reach the same conclusion.

\section{Conclusions}

We have analysed the effect of the compression of the dark matter due to the infall of baryons to the galactic center on the gamma-ray flux. In addition, we have also consider the effect of non-universal soft terms. This analysis shows that neutralino dark matter annihilation can give rise to signals largely reachable by future experiments like GLAST. In particular, we observe that, basically, the whole parameter space of general SUGRA will be tested by GLAST. Even for the mSUGRA case, points corresponding to a value of $\tan \beta$ as small as 5 will be tested. This is a remarkable result if we realise that direct detection experiments will only be able to cover a small region of the parameter space. In contrast, we have observed that indirect detection experiments will be able to test the parameter space of SUGRA. Let us point out that in this analysis we have taken into account all the recent experimental constraints, such as the lower bound on the Higgs mass, the $b \rightarrow s \gamma$ branching ratio, the muon $g-2$ and the recently improved upper bound on $B\left(B_{s} \rightarrow \mu^{+} \mu^{-}\right)$, as well as the astrophysical (WMAP) bounds on the dark matter density.

Actually, in this SUGRA framework we have also been able to fit present excess from EGRET and CANGAROO using different non-universal scenarios, and even fit the data from both experiments with only one scenario. We have also studied the recent HESS data implying a neutralino heavier than $12 \mathrm{TeV}$. Because of such a heavy neutralino, it is not natural to find solutions in the SUGRA framework. Nevertheless we have carried out a quite model-independent analysis, and found the conditions required on the particle physics side to fit the HESS data thanks to dark matter annihilation.

In any case, we must keep in mind that the current data obtained by the different gamma-rays observations from the galactic center region by these three experiments do not allow us to conclude about a dark matter annihilation origin rather than other, less exotic astrophysics sources. Fortunately, this situation may change with the improvement of angular resolution and energy sensitivity of future detectors like GLAST, which will be a complementary source of gamma-ray data.

\section{Acknowledgements}

Y. Mambrini thanks specially J. Paul for useful discussions. E. Nezri warmly thanks C. Woodswolf-Castanier for his essential help and F.-S. Ling for usefull discussions. The work of Y. Mambrini was financially supported by the DESY Theory Group. E. Nezri work was supported by the I.I.S.N. and the Belgian Federal Science Policy (return grant and IAP 5/27). The work of C. Muñoz was supported in part by the Spanish DGI of the MEC under Proyectos Nacionales BFM2003-01266 and FPA2003-04597; 
also by the European Union under the RTN program MRTN-CT-2004-503369, and under the ENTApP Network of the ILIAS project RII3-CT-2004-506222; and also by KAIST under the Visiting Professor Program.

\section{References}

[1] For reviews, see e.g. M. Persic, P. Salucci and F. Stel, 'The universal rotation curve of spiral galaxies: I. The dark matter connection', Mon. Not. Roy. Astron. Soc. 281 (1996) 27 [arXiv:astro-ph/9506004];

Y. Sofue and V. Rubin, 'Rotation curves of spiral galaxies', [arXiv:astroph/0010594];

M. Roncadelli, 'Searching for dark matter', [arXiv:astro-ph/0307115];

P. Salucci and M. Persic, 'Dark matter halos around galaxies', [arXiv:astro$\mathrm{ph} / 9703027]$

E. Battaner and E. Florido, 'The rotation curve of spiral galaxies and its cosmological implications', Fund. Cosmic Phys. 21 (2000) 1 [arXiv:astro-ph/0010475].

[2] For a review, see W. L. Freedman, 'Determination of cosmological parameters', Phys. Scripta T85 (2000) 37 [arXiv:astro-ph/9905222].

[3] WMAP Collaboration, C. L. Bennett et al., 'First year wilkinson microwave anisotropy probe (WMAP) observations: preliminary maps and basic results', Astrophys. J. Suppl. 148 (2003) 1 [arXiv:astro-ph/0302207]; D. N. Spergel et al., 'First year wilkinson microwave anisotropy probe (WMAP) observations: determination of cosmological parameters', Astrophys. J. Suppl. 148 (2003) 175 [arXiv:astro-ph/0302209]; L. Verde et al., 'First year Wilkinson microwave anisotropy probe (WMAP) observations: parameter estimation methodology', Astrophys. J. Suppl. 148 (2003) 195 [arXiv:astro-ph/0302218].

[4] J. E. Gunn, B. W. Lee, I. Lerche, D. N. Schramm and G. Steigman, 'Some astrophysical consequences of the existence of a heavy stable neutral lepton', Astrophys. J. 223 (1978) 1015;

F. W. Stecker, 'The cosmic gamma-ray background from the annihilation of primordial stable neutral heavy leptons', Astrophys. J. 223 (1978) 1032;

Y. Zeldovich, A. Klypin, M. Khlopov and V. Chechetkin 'Astrophysical bounds on the mass of heavy stable neutral leptons', Yadernaya Fizika (1980) 31, 1286.

[English translation: Sov. J. Nucl. Phys. (1980) 31, 664];

M. Srednicki, S. Theisen and J. Silk, 'Cosmic quarkonium: A probe of dark matter' Phys. Rev. Lett. 56 (1986) 263, Erratum-ibid. 56 (1986) 1883;

S. Rudaz, 'Cosmic production of quarkonium?', Phys. Rev. Lett. 56 (1986) 2128;

M. S. Turner, 'Probing the structure of the galactic halo with gamma rays produced by annihilations of weakly interacting massive particle', Phys. Rev. D34 (1986) 34;

L. Bergstrom and H. Snellman, 'Observable monochromatic photons from cosmic photino annihilation', Phys. Rev. D37 (1988) 3737; 
F. W. Stecker, Phys. Lett. B201 (1988) 529;

F. W. Stecker and A. J. Tylka, 'Spectra, fluxes and observability of gamma-rays from dark matter annihilation in the galaxy', Astrophys. J. 343 (1989) 169;

A. Bouquet, P. Salati and J. Silk, 'Gamma-ray lines as a probe for a cold dark matter halo', Phys. Rev. D40 (1989) 3168;

S. Rudaz and F. W. Stecker, 'On the observability of the gamma-ray line flux from dark matter annihilation' Astrophys. J. 368 (1991) 406.

[5] M. Urban, A. Bouquet, B. Degrange, P. Fleury, J. Kaplan, A. L. Melchior and E. Pare, 'Searching for TeV dark matter by atmospheric Cherenkov techniques', Phys. Lett. B293 (1992) 136 [arXiv:hep-ph/9208255];

V. S. Berezinsky, A. V. Gurevich and K. P. Zybin, 'Distribution of dark matter in the galaxy and the lower limits for the masses of supersymmetric particles', Phys. Lett. B294 (1992) 221;

V. Berezinsky, A. Bottino and G. Mignola, 'High-energy gamma radiation from the galactic center due to neutralino annihilation', Phys. Lett. B325 (1994) 136 [arXiv:hep-ph/9402215].

[6] L. Bergstrom, J. Edsjo and P. Ullio, 'Possible indications of a clumpy dark matter halo', Phys. Rev. D58 (1998) 083507 [arXiv:astro-ph/9804050];

L. Bergstrom, J. Edsjo, P. Gondolo and P. Ullio, 'Clumpy neutralino dark matter', Phys. Rev. D59 (1999) 043506 [arXiv:astro-ph/9806072].

[7] J. L. Feng, K. T. Matchev, F. Wilczek, 'Prospects for indirect detection of neutralino dark matter', Phys. Rev. D63 (2001) 045024 [arXiv:astro-ph/0008115].

[8] P. Ullio, L. Bergstrom, J. Edsjo and C. Lacey, 'Cosmological dark matter annihilations into gamma-rays - a closer look', Phys. Rev. D66 (2002) 123502 [astroph/0207125].

[9] L. Bergstrom, P. Ullio and J. H. Buckley, 'Observability of gamma rays from dark matter neutralino annihilations in the Milky Way halo', Astropart. Phys. 9 (1998) 137 [arXiv:astro-ph/9712318].

[10] L. Bergstrom and P. Ullio, 'Full one-loop calculation of neutralino annihilation into two photons', Nucl. Phys. B504 (1997) 27 [arXiv:hep-ph/9706232];

Z. Bern P. Gondolo and M. Perelstein, 'Neutralino annihilation into two photons', Phys. Lett. B411 (1997) 86 [arXiv:hep-ph/9706538].

[11] P. Ullio and L. Bergstrom, 'Neutralino annihilation into a photon and a $Z$ boson', Phys. Rev. D57 (1998) 1962 [arXiv:hep-ph/9707333].

[12] V. S. Berezinsky, A. Bottino and V. de Alfaro, 'Is it possible to detect the gamma ray line from neutralino-neutralino annihilation?', Phys. Lett. B274 (1992) 122.

[13] H. U. Bengtsson, P. Salati and J. Silk, 'Quark flavours and the $\gamma$-ray spectrum from halo dark matter annihilations', Nucl. Phys., B346 (1990) 129.

[14] G. Bertone, G. Servant and G. Sigl, 'Indirect detection of Kaluza-Klein dark matter', Phys. Rev. D68 (2003) 044008 [arXiv:hep-ph/0211342]. 
[15] A. Cesarini, F. Fucito, A. Lionetto, A. Morselli and P. Ullio, 'The galactic center as a dark matter gamma-ray source', Astropart. Phys. 21 (2004) 267 [arXiv:astroph/0305075].

[16] W. de Boer, M. Herold, C. Sander and V. Zhukov, 'Indirect evidence for the supersymmetric nature of dark matter from the combined data on galactic positrons, antiprotons and gamma rays', arXiv:hep-ph/0309029.

[17] D. Hooper and L.-T. Wang, 'Direct and indirect detection of neutralino dark matter in selected supersymmetry breaking scenarios', Phys. Rev. D69 (2004) 035001 [arXiv:hep-ph/0309036].

[18] H. Baer, A. Belyaev, T.Krupovnickas and J. O'Farrill, 'Indirect, direct and collider detection of neutralino dark matter', JCAP 08 (2004) 005 [arXiv:hep-ph/0405210].

[19] J. Edsjo, M. Schelke and P. Ullio, 'Direct versus indirect detection in mSUGRA with self-consistent halo models', arXiv:astro-ph/0405414.

[20] D. Hooper and B. Dingus, 'Improving the angular resolution of EGRET and new limits on supersymmetric dark matter near the galactic center', arXiv:astro$\mathrm{ph} / 0212509$.

[21] A. Bottino, F. Donato, N. Fornengo and S. Scopel, 'Indirect signals from light neutralinos in supersymmetric models without gaugino mass unification', Phys. Rev. D70 (2004) 015005 [arXiv:hep-ph/0401186].

[22] D. Elsasser and K. Mannheim, 'Supersymmetric dark matter and the extragalactic gamma ray background', Phys. Rev. Lett. 94 (2005) 171302 [arXiv:astro$\mathrm{ph} / 0405325]$.

[23] G. Bertone, E. Nezri, J. Orloff and J. Silk, Phys. Rev. D70, 063503 (2004) [arXiv:astro-ph/0403322].

[24] G. Bertone, P. Binetruy, Y. Mambrini and E. Nezri, 'Annihilation radiation of dark matter in heterotic orbifold models', arXiv:hep-ph/0406083.

[25] P. Binetruy, A. Birkedal-Hansen, Y. Mambrini and B.D. Nelson, 'Phenomenological aspects of heterotic orbifold models at one loop', arXiv:hep-ph/0308047.

[26] Y. Mambrini and C. Muñoz, 'Gamma-ray detection from neutralino annihilation in non-universal SUGRA scenarios', arXiv:hep-ph/0407158.

[27] H. Baer, A. Mustafayev, S. Profumo, A. Belyaev, X. Tata, 'Direct, Indirect and Collider Detection of Neutralino Dark Matter In Susy Models with Non-universal Higgs Masses', arXiv:hep-ph/0504001.

[28] Y. Mambrini and C. Muñoz, 'A comparison between direct and indirect dark matter search', JCAP 10 (2004) 003 [arXiv:hep-ph/0407352].

[29] W. de Boer, M. Herold, C. Sander, V. Zhukov, A.V. Gladyshev and D.I. Kazakov, 'Excess of EGRET galactic gamma ray data interpreted as dark matter annihilation', arXiv:astro-ph/0408272;

W. de Boer, 'Evidence for dark matter annihilation from galactic gamma rays?', arXiv:hep-ph/0408166. 
[30] K. Belotsky, D. Fargion, M. Khlopov and R. Konoplich, 'May heavy neutrinos solve underground and cosmic ray puzzles?', arXiv:hep-ph/0411093.

[31] EGRET Collaboration, S. D. Hunger et al., 'EGRET observations of the diffuse gamma-ray emission from the galactic plane', Astrophys. J. 481 (1997) 205; H. A. Mayer-Hasselwander et al., 'High-Energy Gamma-Ray Emission from the Galactic Center' Astron. \& Astrophys. 335 (1998) 161.

[32] CANGAROO-II Collaboration, K. Tsuchiya et al., 'Detection of sub-TeV gammarays from the galactic center direction by CANGAROO-II', Astrophys. J. 606 (2004) L115 [arXiv:astro-ph/0403592].

[33] HESS Collaboration, F. Aharonian et al., 'Very high energy gamma rays from the direction of Sagittarius $A^{*}$, Astron. 83 Astrophys. L13 (2004) 425 [arXiv:astro$\mathrm{ph} / 0408145]$.

[34] N. Gehrels, P. Michelson, 'GLAST: the next generation high-energy gamma-ray astronomy mission', Astropart. Phys. 11 (1999) 277;

See also the web page http://www-glast.stanford.edu

[35] For a recent review, see C. Muñoz, 'Dark matter detection in the light of recent experimental results', Int. J. Mod. Phys. A19 (2004) 3093 [arXiv:hep-ph/0309346].

[36] F. Prada, A. Klypin, J. Flix, M. Martinez and E. Simonneau, 'Astrophysical inputs on the SUSY dark matter annihilation detectability', arXiv:astro-ph/0401512.

[37] J. F. Navarro, C. S. Frenk and S. D. M. White, 'The structure of cold dark matter halos', Astrophys. J. 462 (1996) 563 [arXiv:astro-ph/9508025].

[38] B. Moore, T. Quinn, F. Governato, J. Stadel and G. Lake, 'Cold collapse and the core catastrophe', Mon. Not. Roy. Astron. Soc. 310 (1999) 1147 [arXiv:astro$\mathrm{ph} / 9903164]$.

[39] S. Baek, Y.G. Kim and P. Ko, 'Neutralino dark matter scattering and $B_{s} \rightarrow \mu^{+} \mu^{-}$ in SUSY models', J. High Energy Phys. 02 (2005) 067 [arXiv:hep-ph/0406033].

[40] S. Baek, D.G. Cerdeño, Y.G. Kim, P. Ko and C. Muñoz, 'Direct detection of neutralino dark matter in supergravity', J. High Energy Phys. 06 (2005) 017 [arXiv:hep-ph/0505019].

[41] L. Hernquist, 'An analitical model for spherical galaxies and bulges', Astrophys. J. 356 (1990) 359.

[42] S.H. Hansen, 'Dark matter density profiles from the Jeans equation', Mon. Not. Roy. Astron. Soc. 352 (2004) L41 [arXiv:astro-ph/0405371].

[43] G. R. Blumenthal, S. M. Faber, R. Flores and J. R. Primack, "Contraction of dark matter galactic halos due to baryonic infall," Astrophys. J. 301 (1986) 27.

[44] R. Jesseit, T. Naab and A. Burkert, "The validity of the adiabatic contraction approximation for dark matter halos" Astrophys. J. Lett. 571 (2002) L89 [arXiv:astro-ph/0204164].

[45] O. Y. Gnedin, A. V. Kravtsov, A. A. Klypin and D. Nagai, "Response of dark matter halos to condensation of baryons: cosmological simulations and improved adiabatic contraction model," Astrophys. J. 616 (2004) 16 [arXiv:astro-ph/0406247]. 
[46] J. Diemand, B. Moore and J. Stadel, 'Convergence and scatter of cluster density profiles', Mon. Not. Roy. Astron. Soc. 353 (2004) 624 [arXiv:astro-ph/0402267].

[47] D. Merrit, 'Evolution of the dark matter distribution at the galactic center,' Phys. Rev. Lett. 92 (2004) 201304.

[48] G. Bertone and D. Merritt, 'Time-dependent models for dark matter at the galactic center', arXiv:astro-ph/0501555.

[49] E. Athanassoula, F. S. Ling and E. Nezri, 'Halo geometry and dark matter annihilation signal', arXiv:astro-ph/0504631.

[50] V. Bertin, E. Nezri and J. Orloff, 'Neutralino dark matter beyond CMSSM universality,' J. High Energy Phys. 02 (2003) 046 [arXiv:hep-ph/0210034].

[51] A. Birkedal-Hansen and B. D. Nelson, 'Relic neutralino densities and detection rates with nonuniversal gaugino masses,' Phys. Rev. D67 (2003) 095006 [arXiv:hep-ph/0211071].

[52] P. Gondolo, J. Edsjo, P. Ullio, L. Bergstrom, M. Schelke and E. A. Baltz, 'DarkSUSY: Computing supersymmetric dark matter properties numerically', arXiv:astro-ph/0406204;

See also the web page http://www.physto.se/〜edsjo/darksusy

[53] A. Djouadi, J. L. Kneur and G. Moultaka, 'SuSpect: a Fortran code for the supersymmetric and Higgs particle spectrum in the MSSM', arXiv:hep-ph/0211331; See also the web page http://www.lpm.univ-montp2.fr:6714/ $\mathrm{kneur/suspect.html}$

[54] V. Bertin, E. Nezri and J. Orloff, Eur. Phys. J. C 26, 111 (2002) [arXiv:hep$\mathrm{ph} / 0204135]$.

[55] Muon g-2 Collaboration, G. W. Bennett et al., 'Measurement of the negative muon anomalous magnetic moment to 0.7-ppm', Phys. Rev. Lett. 92 (2004) 161802 [arXiv:hep-ex/0401008].

[56] M. Davier, S. Eidelman, A. Hocker and Z. Zhang, 'Updated estimate of the muon magnetic moment using revised results from $e^{+} e^{-}$annihilation', Eur. Phys. J. C 31 (2003) 503 [arXiv:hep-ph/0308213];

K. Hagiwara, A. D. Martin, D. Nomura and T. Teubner, 'Predictions for $g-2$ of the muon and $\alpha_{Q E D}\left(M_{Z}^{2}\right)^{\prime}$, Phys. Rev. D69 (2004) 093003 [arXiv:hep-ph/0312250];

J. F. de Troconiz and F. J. Yndurain, 'The hadronic contributions to the anomalous magnetic moment of the muon', [arXiv:hep-ph/0402285].

[57] CLEO Collaboration, S. Chen et al., 'Branching fraction and photon energy spectrum for $b \rightarrow s \gamma^{\prime}$, Phys. Rev. Lett. 87 (2001) 251807 [arXiv:hep-ex/0108032].

[58] BELLE Collaboration, H. Tajima, 'Belle B physics results', Int. J. Mod. Phys. A17 (2002) 2967 [arXiv:hep-ex/0111037].

[59] G. Belanger, F. Boudjema, A. Pukhov and A. Semenov, 'micrOMEGAs: a program for calculating the relic density in the MSSM', Comput. Phys. Commun. 149 (2002) 103 [arXiv:hep-ph/0112278];

G. Belanger, F. Boudjema, A. Pukhov and A.G. Semenov, 'MicrOMEGAs: Version 1.3', [arXiv:hep-ph/0405253]; 
See also the web page http://wwwlapp.in2p3.fr/lapth/micromegas

[60] CDF Collaboration, D. Acosta et al., 'Search for $B_{s}^{0} \rightarrow \mu^{+} \mu^{-}$and $B_{d}^{0} \rightarrow \mu^{+} \mu^{-}$ decays in $p \bar{p}$ collisions at $\sqrt{s}=1.96$ TeV', Phys. Rev. Lett. 93 (2004) 032001 [arXiv:hep-ex/0403032];

D0 Collaboration, V. M. Abazov et al., 'A search for the flavor-changing neutral current decay $B_{s}^{0} \rightarrow \mu^{+} \mu^{-}$in $p \bar{p}$ collisions at $\sqrt{s}=1.96 \mathrm{TeV}$ with the D0 detector', Phys. Rev. Lett. 94 (2005) 071802 [arXiv:hep-ex/0410039].

[61] I. Busching, M. Pohl and R. Schlickeiser, 'Excess GeV radiation and cosmic ray origin', Astron. $\&$ Astrophys. 377 (2001) 1056 [arXiv:astro-ph/0108321];

A. D. Erlykin and A. W. Wolfendale, 'Supernova remnants and the origin of cosmic radiation: evidence from low-energy gamma-rays', J. Phys. G28 (2002) 2329;

F. A. Aharonian and A. M. Atoyan, 'Broad-band diffuse gamma-ray emission of the galactic disk', Astron. \& Astrophys. 362 (2000) 937 [arXiv:astro-ph/0009009];

A. W. Strong, I. V. Moskalenko and O. Reimer, 'Diffuse galactic continuum gamma rays. A model compatible with EGRET data and cosmic-ray measurements', Astrophys. J. 613 (2004) 962 [arXiv:astro-ph/0406254].

T. Kamae, T. Abe and T. Koi, 'Diffractive interaction and scaling violation in $p p \rightarrow \pi^{0}$ interaction and $\mathrm{GeV}$ excess in galactic diffuse gamma-ray spectrum of EGRET', arXiv:astro-ph/0410617.

[62] W. de Boer, 'Indirect evidence for WIMP annihilation from diffuse galactic gamma rays', arXiv:astro-ph/0412620.

[63] D. Hooper, I. de la Calle Perez, J. Silk, F. Ferrer and S. Sarkar, 'Have atmospheric Cherenkov telescopes observed dark matter?', J. Cosm. Astrop. Phys. 0409 (2004) 002 [arXiv:astro-ph/0404205].

[64] G. Belanger, F. Boudjema, A. Cottrant, A. Pukhov and A. Semenov, 'WMAP constraints on SUGRA models with non-universal gaugino masses and prospects for direct detection', Nucl. Phys. B706 (2005) 411 [arXiv:hep-ph/0407218].

[65] N. Fornengo, L. Pieri and S. Scopel, 'Neutralino annihilation into gammarays in the Milky Way and in external galaxies', Phys. Rev. D70 (2004) 103529 [arXiv:hep-ph/0407342].

[66] T. Sjostrand et al., 'High-Energy-Physics Event Generation with PYTHIA 6.1', Comput. Phys. Commun. 135 (2001) 238 [arXiv:hep-ph/0010017]. 